\title{
Colchiceine Complexes with Lithium, Sodium and Potassium Salts - Spectroscopic Studies
}

\author{
Joanna Kurek, * Piotr Barczyński
}

\begin{abstract}
Faculty of Chemistry, Laboratory of Chemistry for Heterocyclic Compounds, A. Mickiewicz University, Umultowska 89b, 61-614 Poznań, Poland
* Corresponding author's e-mail address: joankur@amu.edu.pl
\end{abstract}

RECEIVED: March 20, 2016 * REVISED: August 19, 2016 * ACCEPTED: August 31, 2016

Abstract: Colchiceine complexes with $\mathrm{Li}^{+}, \mathrm{Na}^{+}$and $\mathrm{K}^{+}$cations have been synthesized and studied by ${ }^{1} \mathrm{H}$ and ${ }^{13} \mathrm{C} \mathrm{NMR}, \mathrm{FT}-\mathrm{IR}, \mathrm{FAB} \mathrm{MS}$ and $\mathrm{UV}-\mathrm{Vis}$. It has been shown that colchiceine forms stable complexes especially with lithium cation and the most stable structures of the complexes are those in which the acetamide groups are involved in the coordination process. The structures of the colchiceine complexes with $\mathrm{Li}^{+}$, $\mathrm{Na}^{+}$and $\mathrm{K}^{+}$ cations are discussed in details.

Keywords: colchiceine, complexes of colchiceine, NMR spectra, FT-IR, FAB MS spectra, UV-Vis spectra.

\section{INTRODUCTION}

$\mathbf{C}^{\circ}$ OLCHICINE 1, in Figure 1, is a tropolone alkaloid of Colchicum autumnale. It shows antimitotic, antifibrotic and anti-inflammatory activity ${ }^{[1]}$ and can efficiently alleviate the symptoms of gout attack when applied in the early phase. More recently, it has been introduced in the

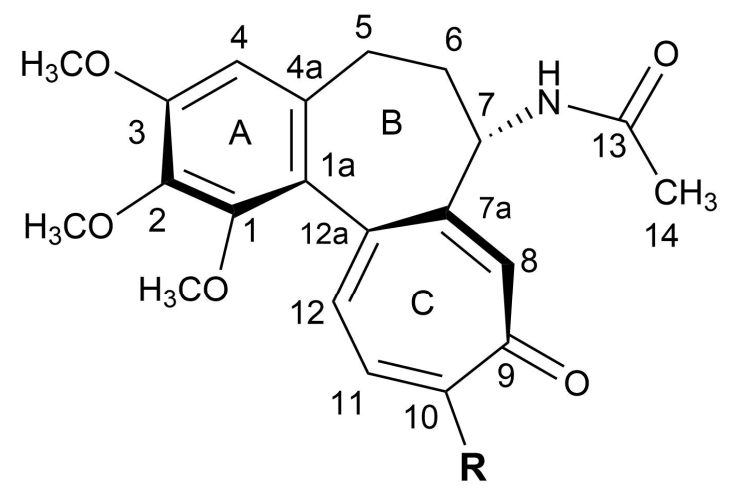

R: $\begin{array}{cc}-\mathrm{OCH}_{3} & 1 \\ -\mathrm{OH} & 2\end{array}$

Figure 1. Structure and carbon atom numbering of Colchicine 1 and Colchiceine 2. treatment of Mediterranean fever. ${ }^{[2]}$ Compound $\mathbf{1}$ is known to be sensitive to light and hydrolysis. ${ }^{[3]}$ Colchiceine 2 (10demetoxy-10-hydroxy-colchicine) in Figure 1 and $\beta$ - and $y$ lumicolchicine are the main degradation products of colchicine.

Compound 2, an important intermediate in the synthesis of colchicine $\mathrm{C}-10$ derivatives, can be obtained from colchicine 1 by a gentle hydrolysis with hydrochloric acid. ${ }^{[4]}$ Colchiceine $\mathbf{2}$ is also a useful reagent for many chemical reactions on ring $\mathrm{C}$, for example tosylation or mesylation to obtain known derivatives or to produce new compounds. Upon tosylation or mesylation, $\mathbf{2}$ is a mixture of two products with substituent at C-9 (biologicallyinactive isocolchicinoids) and C-10 (biologically-active colchicinoids) carbon atom, is caused by tosyl group migration. ${ }^{[5-7]}$

It is necessary for biologically active compounds to be easily analysed and detected by analytical methods, for example TLC and HPLC. HPLC analyses of $\mathbf{2}$ turned out to be more difficult than of $\mathbf{1}$ and Colchicum plant extracts. Colchiceine can be present in plant extracts besides colchicine, or can appear as a degradation product on storage of 1. ${ }^{[8-11]}$ Colchiceine can easily form complexes with most of silica based phases, which can be explained by the properties of its ring system especially ring $\mathrm{C}$ with a 
hydroxyl group at C-10 position and neighbouring carbonyl group at carbon atom C-9. It is difficult to detect and separate colchiceine from plant extracts because of its chelating properties. To extensively recovery of colchiceine from silica column matrixes in a HPLC analysis a suitable metal cation in salt form, which can form complexes with $\mathbf{2}$ was added. For better separation of $\mathbf{2}$ from a mixture of alkaloids in plant extracts, usually inorganic salts are added. For instance to aqueous solution of $\mathbf{2}$, ferric chloride is added to get a coloured complex, then the solution is extracted with chloroform. In the HPLC analysis copper sulphate usually was added to the mobile phase to form coloured complexes of metal-colchiceine. $[3,12,13]$

After administration of colchicine, colchiceine has been reported as a metabolite in rats, ${ }^{[14]}$ but it has not been demonstrated to occur in humans. ${ }^{[15]}$ Mourelle et al. have proposed that colchiceine acts as an antioxidant and protective agent against lipid peroxidation in a rat model of liver injury. ${ }^{[16]}$ 10-Demetoxy-10-hydroxy-colchicine (colchiceine) has not been confirmed to occur in humans, but is produced in rodents by the same CYP homolog. ${ }^{[17,18]}$ In a biochemical model of liver injury, $\mathbf{2}$ is the metabolite, suggested to have beneficial effects. ${ }^{[19]}$ It has been shown that $\mathbf{2}$ prevents acute and chronic $\mathrm{CCl}_{4}$ injury in rats. ${ }^{[15,20]}$ Furthermore in an earlier study, $\mathbf{2}$ has been shown to prevent lipid peroxidation the centerpiece of $\mathrm{CCl}_{4}$-induced injury. Protection against lipid peroxidation has also been observed in a rat model of biliary obstruction in which $\mathbf{2}$ was compared with vitamin E. ${ }^{[21]}$ High concentrations of colchiceine has also been shown to protect reduced glutathione (GSH) against oxidation by atmospheric oxygen and to prevent oxygen-induced inactivation of enzymes with catalytically essential sulfhydryl groups. ${ }^{[22]}$ In vivo experiments have suggested that $\mathbf{2}$ acts as an effective antioxidant at pharmacologically relevant concentrations, ${ }^{[21]}$ although direct evidence for $\mathbf{2}$ radical scavenging activity and protection against lipid peroxidation is lacking. ${ }^{[15]}$

Moreover, colchiceine has been demonstrated to be less toxic than colchicine in the evaluation of the effect on cytochrome P450 (CYP) expression. It has been concluded that colchiceine is not cytotoxic in primary human hepatocytes.[23] Interestingly, colchiceine has better antifibrotic properties than colchicine, it can be used as a $\mathbf{1}$ substitute with anticipated fewer side-effects.[20] Colchiceine, a closely related structural analogue of colchicine with a C-ring tropolone, has been shown to be a potent inhibitor of microtubule assembly in vitro. The mechanism of inhibition is mediated through binding to tubulin although potentially not through the colchicine receptor site.[24]

The formation of complexes between colchicines and cations has not been the subject of great interest. Only
Mackay et al. in 1998 obtained hydrated crystals of copper(II) colchiceine, belonging to the tetragonal space group. ${ }^{[16]}$ In this structure the tropolonic oxygens, from two colchiceine molecules, are coordinated to the copper atom in this bis-chelated complex to form a square planar arrangement. The positions of three water molecules are clearly defined. An intricate hydrogen-bonding system links the complex and water molecules into a three-dimensional network in the crystal..16]

In the previous work we have reported the coordination process of colchicine with iodides and perchlorates monovalent metal ions (lithium, sodium and potassium salts). ${ }^{[25]}$ Although colchicine is a very important commercially available alkaloid, its complexes (except in Ref. 25) and complexes of colchicine derivatives like colchiceine have not been thoroughly characterized yet.

The complexation process of colchiceine with monovalent metal ions has not been studied yet. This fact has prompted us to obtain and examine colchiceine complexes 3-8 with monovalent cations: $\mathrm{Li}^{+}, \mathrm{Na}^{+}$and $\mathrm{K}^{+}$. Molecular structures for all obtained colchiceine complexes were established by of $1 \mathrm{D}$ and $2 \mathrm{D} \mathrm{NMR,} \mathrm{IR,} \mathrm{UV} \mathrm{and} \mathrm{mass}$ spectral analysis. From the practical point of view this process of complexation can be useful for isolation colchicines from plant extracts.

\section{EXPERIMENTAL}

\section{Material}

Colchiceine was synthesized from colchicine (Aldrich) by acidic hydrolysis in the mild conditions. ${ }^{[26]}$ Obtained colchiceine is a monohydrate compound. The colchiceine complexes 3-8 with salts $\left(\mathrm{LiClO}_{4}\right.$, $\mathrm{Lil}, \mathrm{NaClO}_{4}, \mathrm{Nal}, \mathrm{KI}, \mathrm{KSCN}$ from Aldrich) were obtained by dissolving of the respective salts and colchiceine in the 1:1 ratio in methanol. Complexes were prepared from colchiceine $(96 \mathrm{mg}, 0,25$ $\mathrm{mmol}$ ) and lithium iodide (33 $\mathrm{mg}, 0,25 \mathrm{mmol})$, lithium perchlorate $(27 \mathrm{mg}, 0.25 \mathrm{mmol})$, sodium iodide $(38 \mathrm{mg}$, $0.25 \mathrm{mmol}$ ), sodium perchlorate $(31 \mathrm{mg}, 0.25 \mathrm{mmol}$ ), potassium iodide $(42 \mathrm{mg}, 0.25 \mathrm{mmol}$ ), potassium thiocyanate $24 \mathrm{mg}, 0.25 \mathrm{mmol}$ ), respectively. Each mixture was stirred for $24 \mathrm{~h}$ in room temperature. The crystalline products were obtained by slow evaporation of the solvent.

Carbon atom numbering of colchicine $\mathbf{1}$ and colchiceine $\mathbf{2}$ is shown in Figure 1.

\section{(3) Complex Colchiceine - Lithium lodide}

103 mg; yield $84 \%$; m.p. $110-112{ }^{\circ} \mathrm{C}$, Anal. Calcd. for $\mathrm{C}_{21} \mathrm{H}_{23} \mathrm{NO}_{6}$. Lil.3.5 $\mathrm{H}_{2} \mathrm{O}: \mathrm{C}, 43.22 ; \mathrm{H}, 5.11 ; \mathrm{N}, 2.41$. Found: $\mathrm{C}$, 43.49; H, 5.31; N, 2.55. IR(KBr) $\tilde{v} / \mathrm{cm}^{-1}: 3411 \mathrm{~m}, 3246 \mathrm{~m}$, 2998 w, 2937 w, 2856 w, 1656 s, 1602 s, 1540 m, 1489 m, $1454 \mathrm{~m}, 1276 \mathrm{~m}, 1195 \mathrm{~s}, 1139 \mathrm{~m}, 483 \mathrm{w} . \mathrm{IR}$ (nujol/fluorolub) $\tilde{v} / \mathrm{cm}^{-1}$ : $3397 \mathrm{~m}, 3243 \mathrm{~m}, 3045 \mathrm{w}, 2952 \mathrm{w}, 2922 \mathrm{w}, 2853 \mathrm{w}$, 2727 w, 1655 s, 1602 s, 1537 m, 1488 m, 1452 m, 1404 w, 
1349 w, 1274 w, 484 w (vs, very strong; s, strong; m, medium; $\mathrm{w}$, weak; and also in further text regarding IR spectra $\left.\left(\tilde{v} / \mathrm{cm}^{-1}\right)\right)$. ${ }^{1 \mathrm{H}} \mathrm{NMR}\left(300 \mathrm{MHz}, \mathrm{DMSO}-d_{6}\right) \delta / \mathrm{ppm}$ : 6.79 (s, HC-4), 2.15 (m, $\left.\mathrm{H}_{2} \mathrm{C}-5\right), 2.55\left(\mathrm{~m}, \mathrm{H}_{2} \mathrm{C}-5\right), 1.94$ (m, $\left.\mathrm{H}_{2} \mathrm{C}-6\right), 2.13$ (m, $\mathrm{H}_{2} \mathrm{C}-6$ ), 4.35 (m, HC-7), 7.30 (s, HC-8), 7.31 (d, $J=11.81 \mathrm{~Hz}, \mathrm{HC}-11), 7.13(\mathrm{~d}, J=11.81 \mathrm{~Hz}, \mathrm{H} \mathrm{C}-12), 3.77$ (s, $\left.\mathrm{CH}_{3} \mathrm{O}-1\right), 3.51$ (s, $\left.\mathrm{CH}_{3} \mathrm{O}-2\right), 3.83$ (s, $\left.\mathrm{CH}_{3} \mathrm{O}-3\right), 1.88$ (s, $\mathrm{H}_{3} \mathrm{C}-$ 14), 8.63 (d, $J=7.14 \mathrm{~Hz}, \mathrm{NH}) .{ }^{13} \mathrm{C}$ NMR $\left(75 \mathrm{MHz}\right.$, DMSO- $\left.d_{6}\right)$

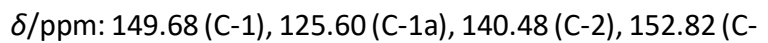
3), 107.70 (C-4), 133.69 (C-4a), 29.24 (C-5), 36.50 (C-6), 51.61 (C-7), 149.94 (C-7a), 123.91 (C-8), 172.00 (C-9), 168.54 (C-10), 118.07 (C-11), 139.91 (C-12), 134.54 (C-12a), $60.71\left(\mathrm{CH}_{3} \mathrm{O}-1\right), 60.69\left(\mathrm{CH}_{3} \mathrm{O}-2\right), 55.87\left(\mathrm{CH}_{3} \mathrm{O}-3\right), 168.90$ (C13), 22.50 (C-14). ${ }^{1} \mathrm{H}$ NMR (300 MHz, $\mathrm{CD}_{3} \mathrm{CN}$ ) $\delta / p p m: 6.74$ (s, HC-4), 2.15 (m, $\left.\mathrm{H}_{2} \mathrm{C}-5\right), 2.55\left(\mathrm{~m}, \mathrm{H}_{2} \mathrm{C}-5\right), 2.09$ (m, $\left.\mathrm{H}_{2} \mathrm{C}-6\right)$, 2.25 (m, H $\left.{ }_{2} \mathrm{C}-6\right), 4.41$ (m, HC-7), 7.51 (s, HC-8), 7.25 (d, $J=$ $7.48 \mathrm{~Hz}, \mathrm{HC}-11$ ), 7.48 (d, J = 7.25 Hz, HC-12), 3.59 (s, $\mathrm{CH}_{3} \mathrm{O}-$ 1), 3.82 (s, $\mathrm{CH}_{3} \mathrm{O}-2$ ), 3.87 (s, $\left.\mathrm{CH}_{3} \mathrm{O}-3\right), 1.94\left(\mathrm{~s}, \mathrm{H}_{3} \mathrm{C}-14\right), 7.65$ (d, $J=4.41 \mathrm{~Hz}, \mathrm{NH}) .{ }^{13} \mathrm{C}$ NMR $\left(75 \mathrm{MHz}, \mathrm{CD}_{3} \mathrm{CN}\right) \delta / \mathrm{ppm}$ : 151.04 (C-1), 126.37 (C-1a), 141.74 (C-2), 154.39 (C-3), 108.44 (C-4), 135.75 (C-4a), 30.03 (C-5), 37.87 (C-6), 53.53 (C-7), 151.31 (C-7a), 124.85 (C-8), 172.94 (C-9), 168.43 (C10), 118.20 (C-11), 142.30 (C-12), 136.24 (C-12a), 61.62 $\left(\mathrm{CH}_{3} \mathrm{O}-1\right), 61.30\left(\mathrm{CH}_{3} \mathrm{O}-2\right), 56.53\left(\mathrm{CH}_{3} \mathrm{O}-3\right), 171.23(\mathrm{C}-13)$, 22.77 (C-14). UV-visible in $\mathrm{CH}_{3} \mathrm{OH} \lambda_{\max } / \mathrm{nm}\left(\varepsilon / \mathrm{mol}^{-1} \mathrm{dm}^{3}\right.$ $\mathrm{cm}^{-1}$ ): 349.5 (17444), 224 (35853).

(4) Complex Colchiceine - Sodium lodide

$131 \mathrm{mg}$; yield $93 \%$; m.p. $98-100{ }^{\circ} \mathrm{C}$, Anal. Calcd. for $\mathrm{C}_{21} \mathrm{H}_{23} \mathrm{NO}_{6} \cdot \mathrm{Nal} \cdot 3 \mathrm{H}_{2} \mathrm{O}: \mathrm{C}, 41.38 ; \mathrm{H}, 4.76 ; \mathrm{N}, 2.29$. Found: $\mathrm{C}$, 41.70; H, 4.71; N, 2.27. IR (KBr) $\tilde{v} / \mathrm{cm}^{-1}: 3436 \mathrm{~m}, 3252 \mathrm{~m}$, 2998 w, 2937 w, 2856 w, 2836 w, 1659 s, 1608 s, 1541 m, 1489 m, 1453 m, 1275 m, 1196 m, 1137 m, 483 w. IR (nujol/fluorolub) ṽ/cm-1: $3407 \mathrm{~m}, 3245 \mathrm{~m}, 3046 \mathrm{~m}, 2953 \mathrm{~m}$, 2937 w, 2855 w, 2728 w, 1659 s, 1606 s, 1534 m, 1489 m, 1455 m, 1404 m, 1349 m, 1276 m, 484 w. ${ }^{1 H}$ NMR (300 MHz, DMSO- $\left.d_{6}\right) \delta / p p m: 6.81$ (s, HC-4), $2.13\left(\mathrm{~m}, \mathrm{H}_{2} \mathrm{C}-5\right), 2.53$ (m, $\left.\mathrm{H}_{2} \mathrm{C}-5\right), 1.97$ ( $\left.m, \mathrm{H}_{2} \mathrm{C}-6\right), 2.11$ ( $\left.m, \mathrm{H}_{2} \mathrm{C}-6\right), 4.34$ (m, HC-7), 7.32 (s, HC-8), 7.34 (d, $J=11.81 \mathrm{~Hz}, \mathrm{HC}-11), 7.16$ (d, $J=11.81$ $\mathrm{Hz}, \mathrm{HC}-12$ ), 3.79 (s, $\left.\mathrm{CH}_{3} \mathrm{O}-1\right)$, 3.53 (s, $\left.\mathrm{CH}_{3} \mathrm{O}-2\right), 3.85$ (s, $\mathrm{CH}_{3} \mathrm{O}-$ 3), $1.88\left(\mathrm{~s}, \mathrm{H}_{3} \mathrm{C}-14\right), 8.65(\mathrm{~d}, J=7.32 \mathrm{~Hz}, \mathrm{NH}) .{ }^{13} \mathrm{C}$ NMR (75 $\left.\mathrm{MHz}, \mathrm{DMSO}-d_{6}\right) \delta / \mathrm{ppm}: 49.75$ (C-1), 125.56 (C-1a), 140.50 (C-2), 152.88 (C-3), 107.74 (C-4), 133.98 (C-4a), 29.24 (C-5), 36.91 (C-6), 51.65 (C-7), 149.95 (C-7a), 124.07 (C-8), 171.74 (C-9), 168.53 (C-10), 118.12 (C-11), 140.00 (C-12), 134.56 (C-12a), $60.74\left(\mathrm{CH}_{3} \mathrm{O}-1\right), 60.72\left(\mathrm{CH}_{3} \mathrm{O}-2\right), 55.91\left(\mathrm{CH}_{3} \mathrm{O}-3\right)$, 168.61 (C-13), 22.51 (C-14). ${ }^{1} \mathrm{H}$ NMR (300 MHz, CD ${ }_{3} \mathrm{CN}$ )

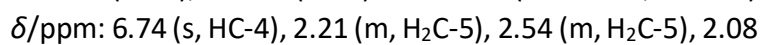
(m, $\left.\mathrm{H}_{2} \mathrm{C}-6\right), 2.25$ (m, $\left.\mathrm{H}_{2} \mathrm{C}-6\right), 4.42$ (m, HC-7), 7.53 (s, HC-8), 7.24 (d, J=7.48 Hz, HC-11), 7.48 (d, J=7.24 Hz, HC-12), 3.60 (s, $\left.\mathrm{CH}_{3} \mathrm{O}-1\right), 3.84$ (s, $\left.\mathrm{CH}_{3} \mathrm{O}-2\right), 3.87$ (s, $\left.\mathrm{CH}_{3} \mathrm{O}-3\right), 1.95$ (s, $\mathrm{H}_{3} \mathrm{C}-$ 14), $7.54(\mathrm{~d}, J=4.42 \mathrm{~Hz}, \mathrm{NH}) .{ }^{13} \mathrm{C}$ NMR $\left(75 \mathrm{MHz}, \mathrm{CD}_{3} \mathrm{CN}\right)$

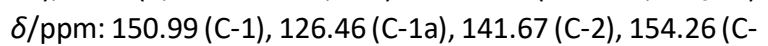
3), 108.40 (C-4), 135.78 (C-4a), 30.05 (C-5), 37.86 (C-6),
53.37 (C-7), 151.30 (C-7a), 124.81 (C-8), 173.06 (C-9), 168.41 (C-10), 117.88 (C-11), 142.13 (C-12), 135.95 (C-12a), $61.53\left(\mathrm{CH}_{3} \mathrm{O}-1\right), 61.30\left(\mathrm{CH}_{3} \mathrm{O}-2\right), 56.50\left(\mathrm{CH}_{3} \mathrm{O}-3\right), 170.42$ (C13), 22.77 (C-14). UV-visible in $\mathrm{CH}_{3} \mathrm{OH} \lambda_{\max } / \mathrm{nm}\left(\varepsilon / \mathrm{mol}^{-1}\right.$ $\mathrm{dm}^{3} \mathrm{~cm}^{-1}$ ): 350 (16762), 223 (36109).

\section{(5) Complex Colchiceine - Potassium lodide} 135 mg; yield $98 \%$; m.p. 94-96 ${ }^{\circ} \mathrm{C}$, Anal. Calcd. for $\mathrm{C}_{21} \mathrm{H}_{23} \mathrm{NO}_{6} \cdot \mathrm{KI} \cdot 2 \mathrm{H}_{2} \mathrm{O}: \mathrm{C}, 42.82 ; \mathrm{H}, 4.56 ; \mathrm{N}, 2.38$. Found: $\mathrm{C}$, 42.72; H, 4.42; N, 2.31. IR (KBr) v/ $/ \mathrm{cm}^{-1}: 3438 \mathrm{~m}, 3359 \mathrm{~m}$, $3128 \mathrm{~m}, 2997 \mathrm{w}, 2938 \mathrm{w}, 2859 \mathrm{w}, 2833 \mathrm{w}, 1653 \mathrm{~s}, 1611 \mathrm{~s}$, 1553 m, 1490 m, 1454 m, 1276 m, 1195 m, 1136 m, 482 w. IR (nujol/fluorolub) ṽ/cm ${ }^{-1}: 3418 \mathrm{~m}, 3244 \mathrm{~m}, 3051 \mathrm{w}, 2953$ w, 2926 w, 2855 w, 2725 w, 1657 s, 1609 s, 1543 m, 1489 m, 1453 m, 1403 m, 1349 m, 1275 m, 484 w. ${ }^{1}$ H NMR (300 $\left.\mathrm{MHz}, \mathrm{DMSO}-d_{6}\right) \delta /$ ppm: 6.81 (s, HC-4), $2.13\left(\mathrm{~m}, \mathrm{H}_{2} \mathrm{C}-5\right), 2.54$ (m, $\left.\mathrm{H}_{2} \mathrm{C}-5\right), 1.98$ (m, $\left.\mathrm{H}_{2} \mathrm{C}-6\right), 2.12$ (m, H2C-6), 4.37 (m, HC7), 7.34 (s, HC-8), 7.36 (d, J=11.81 Hz, HC-11), 7.18 (d, $J=$ $11.81 \mathrm{~Hz}, \mathrm{HC}-12), 3.80$ (s, $\left.\mathrm{CH}_{3} \mathrm{O}-1\right), 3.54\left(\mathrm{~s}, \mathrm{CH}_{3} \mathrm{O}-2\right), 3.85$ (s, $\left.\mathrm{CH}_{3} \mathrm{O}-3\right), 1.89$ (s, $\left.\mathrm{H}_{3} \mathrm{C}-14\right), 8.65(\mathrm{~d}, J=7.31 \mathrm{~Hz}, 1 \mathrm{H}-\mathrm{NH}) .{ }^{13} \mathrm{C}$

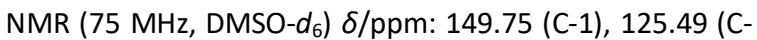
1a), 140.46 (C-2), 152.85 (C-3), 107.71 (C-4), 134.07 (C-4a), 29.19 (C-5), 36.88 (C-6), 51.62 (C-7), 149.91 (C-7a), 124.07 (C-8), 171.57 (C-9), 168.32 (C-10), 118.08 (C-11), 139.99 (C12), 134.52 (C-12a), $60.70\left(\mathrm{CH}_{3} \mathrm{O}-1\right), 60.67\left(\mathrm{CH}_{3} \mathrm{O}-2\right), 55.88$ $\left(\mathrm{CH}_{3} \mathrm{O}-3\right), 168.53$ (C-13), 22.48 (C-14). ${ }^{1 \mathrm{H}} \mathrm{NMR}(300 \mathrm{MHz}$, $\mathrm{CD}_{3} \mathrm{CN}$ ) $\delta / \mathrm{ppm}: 6.74$ (s, HC-4), $2.21\left(\mathrm{~m}, \mathrm{H}_{2} \mathrm{C}-5\right), 2.55\left(\mathrm{~m}, \mathrm{H}_{2} \mathrm{C}-\right.$ 5), $2.03\left(\mathrm{~m}, \mathrm{H}_{2} \mathrm{C}-6\right), 2.19\left(\mathrm{~m}, \mathrm{H}_{2} \mathrm{C}-6\right), 4.42$ (m, HC-7), 7.52 (s, HC-8), 7.25 (d, J = 7.48 Hz, HC-11), 7.48 (d, J=7.25 Hz, HC12), 3.61 (s, $\left.\mathrm{CH}_{3} \mathrm{O}-1\right), 3.84$ ( $\left.\mathrm{s}, \mathrm{CH}_{3} \mathrm{O}-2\right), 3.87$ ( $\left.\mathrm{s}, \mathrm{CH}_{3} \mathrm{O}-3\right), 1.94$ (s, $\left.\mathrm{H}_{3} \mathrm{C}-14\right), 7.44$ (d, $\left.J=4.42 \mathrm{~Hz}, \mathrm{NH}\right) .{ }^{13} \mathrm{C}$ NMR $(75 \mathrm{MHz}$, $\mathrm{CD}_{3} \mathrm{CN}$ ) $\delta / \mathrm{ppm}: 151.00$ (C-1), 126.48 (C-1a), 141.70 (C-2), 154.30 (C-3), 108.41 (C-4), 135.78 (C-4a), 30.06 (C-5), 37.86 (C-6), 53.35 (C-7), 151.29 (C-7a), 124.75 (C-8), 173.09 (C-9), 168.24 (C-10), 117.66 (C-11), 142.12 (C-12), 135.94 (C-12a), $61.49\left(\mathrm{CH}_{3} \mathrm{O}-1\right), 61.28\left(\mathrm{CH}_{3} \mathrm{O}-2\right), 56.49\left(\mathrm{CH}_{3} \mathrm{O}-3\right), 170.17$ (C13), 22.74 (C-14). UV-visible in $\mathrm{CH}_{3} \mathrm{OH} \lambda_{\max } / \mathrm{nm}\left(\varepsilon / \mathrm{mol}^{-1}\right.$ $\mathrm{dm}^{3} \mathrm{~cm}^{-1}$ ): 350.5 (17292), 222.5 (39813).

(6) Complex Colchiceine - Lithium Perchlorate 114 mg; yield $94 \%$; m.p. $100-102{ }^{\circ} \mathrm{C}$, Anal. Calcd. for $\mathrm{C}_{21} \mathrm{H}_{23} \mathrm{NO}_{6} \cdot \mathrm{LiClO}_{4} \cdot 2.5 \mathrm{H}_{2} \mathrm{O}: \mathrm{C}, 46.93 ; \mathrm{H}, 5.21 ; \mathrm{N}, 2.61$. Found: $\mathrm{C}, 46.54 ; \mathrm{H}, 5.11 ; \mathrm{N}, 2.55$. IR (KBr) v $/ \mathrm{cm}^{-1}: 3394 \mathrm{~m}, 3245 \mathrm{~m}$, 3059 w, 3000 w, 2939 w, 2859 w, 2838 w, 1654 s, 1606 s, 1543 m, 1489 m, 1456 m, 1277 m, 1196 m, 1143 m, 1087 vs, $1044 \mathrm{~m}, 918 \mathrm{w}, 625 \mathrm{w}, 484 \mathrm{w}$. IR (nujol/fluorolub) $\tilde{v} / \mathrm{cm}^{-1}: 3573 \mathrm{~m}, 3526 \mathrm{~m}, 3366 \mathrm{~m}, 2951 \mathrm{w}, 2925 \mathrm{w}, 2855 \mathrm{w}$, $2726 \mathrm{w}, 1629 \mathrm{~s}, 1611 \mathrm{~s}, 1552 \mathrm{~m}, 1491 \mathrm{~m}, 1456 \mathrm{~m}, 1404 \mathrm{~m}$, 1349 m, 1278 m, 1094 vs, 1011 m, 920 w, 625 w, 483 w. ${ }^{1} \mathrm{H}$

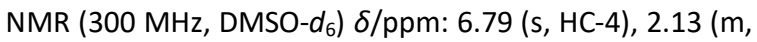
$\left.\mathrm{H}_{2} \mathrm{C}-5\right), 2.55\left(\mathrm{~m}, \mathrm{H}_{2} \mathrm{C}-5\right), 1.94\left(\mathrm{~m}, \mathrm{H}_{2} \mathrm{C}-6\right), 2.11\left(\mathrm{~m}, \mathrm{H}_{2} \mathrm{C}-6\right)$, 4.34 (m, HC-7), 7.31 (s, HC-8), 7.33 (d, J = $11.81 \mathrm{~Hz}, \mathrm{HC}-11$ ), $7.15(\mathrm{~d}, J=11.81 \mathrm{~Hz}, \mathrm{HC}-12), 3.77\left(\mathrm{~s}, \mathrm{CH}_{3} \mathrm{O}-1\right), 3.51\left(\mathrm{~s}, \mathrm{CH}_{3} \mathrm{O}-\right.$ 2), $3.83\left(\mathrm{~s}, \mathrm{CH}_{3} \mathrm{O}-3\right), 1.88$ (s, $\left.\mathrm{H}_{3} \mathrm{C}-14\right), 8.64$ (d, J= $7.42 \mathrm{~Hz}$, $\mathrm{NH}) .{ }^{13} \mathrm{C}$ NMR $\left(75 \mathrm{MHz}, \mathrm{DMSO}-d_{6}\right) \delta / \mathrm{ppm}: 149.82$ (C-1), 
125.59 (C-1a), 140.55 (C-2), 152.95 (C-3), 107.76 (C-4), 134.14 (C-4a), 29.29 (C-5), 36.97 (C-6), 51.69 (C-7), 150.02 (C-7a), 124.18 (C-8), 171.71 (C-9), 168.47 (C-10), 118.16 (C11), 140.09 (C-12), 134.63 (C-12a), $60.80\left(\mathrm{CH}_{3} \mathrm{O}-1\right), 60.77$ ( $\left.\mathrm{CH}_{3} \mathrm{O}-2\right), 55.91\left(\mathrm{CH}_{3} \mathrm{O}-3\right), 168.69$ (C-13), 22.55 (C-14). ${ }^{1} \mathrm{H}$ NMR ( $300 \mathrm{MHz}, \mathrm{CD}_{3} \mathrm{CN}$ ) $\delta / \mathrm{ppm}: 6.74$ (s, HC-4), $2.22\left(\mathrm{~m}, \mathrm{H}_{2} \mathrm{C}-\right.$ 5), $2.56\left(m, \mathrm{H}_{2} \mathrm{C}-5\right), 1.96\left(\mathrm{~m}, \mathrm{H}_{2} \mathrm{C}-6\right), 2.26\left(\mathrm{~m}, \mathrm{H}_{2} \mathrm{C}-6\right), 4.37$ (m, HC-7), 7.46 (s, HC-8), 7.26 (d, J = 7.48 Hz, HC-11), 7.48 (d, $J=7.25 \mathrm{~Hz}, \mathrm{HC}-12), 3.60$ (s, $\left.\mathrm{CH}_{3} \mathrm{O}-1\right), 3.83$ (s, $\left.\mathrm{CH}_{3} \mathrm{O}-2\right)$, 3.87 (s, $\mathrm{CH}_{3} \mathrm{O}-3$ ), 1.92 (s, $\left.\mathrm{H}_{3} \mathrm{C}-14\right), 7.48$ (d, J = 4.37 Hz, NH). ${ }^{13} \mathrm{C}$ NMR $\left(75 \mathrm{MHz}, \mathrm{CD}_{3} \mathrm{CN}\right.$ ) $\delta / \mathrm{ppm}$ : 151.08 (C-1), 126.41 (C1a), 141.78 (C-2), 154.45 (C-3), 108.48 (C-4), 135.81 (C-4a), 30.06 (C-5), 37.91 (C-6), 53.49 (C-7), 151.19 (C-7a), 125.07 (C-8), 172.98 (C-9), 168.48 (C-10), 117.95 (C-11), 142.36 (C-12), 136.29 (C-12a), $61.65\left(\mathrm{CH}_{3} \mathrm{O}-1\right), 61.35\left(\mathrm{CH}_{3} \mathrm{O}-2\right)$, $56.54\left(\mathrm{CH}_{3} \mathrm{O}-3\right), 170.08$ (C-13), 22.69 (C-14). UV-visible in $\mathrm{CH}_{3} \mathrm{OH} \lambda_{\max } / \mathrm{nm}\left(\varepsilon / \mathrm{mol}^{-1} \mathrm{dm}^{3} \mathrm{~cm}^{-1}\right): 348.5$ (16192), 231 (27017).

\section{(7) Complex Colchiceine - Sodium Perchlorate}

125 mg; yield $93 \%$; m.p. $102-104{ }^{\circ} \mathrm{C}$, Anal. Calcd. for $\mathrm{C}_{21} \mathrm{H}_{23} \mathrm{NO}_{6} \cdot \mathrm{NaClO}_{4} \cdot 2 \mathrm{H}_{2} \mathrm{O}: \mathrm{C}, 47.27 ; \mathrm{H}, 4.95 ; \mathrm{N}, 2.57$. Found: C, 47.24; H, 4.87; N, 2.52. IR (KBr) $\tilde{v} / \mathrm{cm}^{-1}: 3364 \mathrm{~m}, 3261$ m, 3063 m, 2998 w, 2940 w, 2858 w, 2839 w, 1662 s, 1611 s, $1544 \mathrm{~m}, 1490 \mathrm{~m}, 1455 \mathrm{~m}, 1276 \mathrm{~m}, 1196 \mathrm{~m}, 1140 \mathrm{~m}$, 1108 m, 1090 vs, 1043 m, 918 w, 626 w, 484 w. IR (nujol/fluorolub) $\tilde{v} / \mathrm{cm}^{-1}: 3538 \mathrm{~m}, 3363 \mathrm{~m}, 3260 \mathrm{~m}, 2996$ w, 2939 w, 2858 w, 2725 w, 1659 s, 1609 s, 1540 m, 1489 m, 1454 m, 1405 m, 1350 m, 1277 m, 1092 vs, 1044 m, $919 \mathrm{w}, 624 \mathrm{w}, 484$ w. ${ }^{1} \mathrm{H}$ NMR (300 MHz, DMSO-d $d_{6}$ ) $\delta / p p m: 6.80$ (s, HC-4), 2.13 (m, $\left.\mathrm{H}_{2} \mathrm{C}-5\right), 2.53\left(\mathrm{~m}, \mathrm{H}_{2} \mathrm{C}-5\right)$, $1.96\left(\mathrm{~m}, \mathrm{H}_{2} \mathrm{C}-6\right), 2.11\left(\mathrm{~m}, \mathrm{H}_{2} \mathrm{C}-5\right), 4.35$ (m, HC-7), 7.33 (s, $\mathrm{HC}-8), 7.35$ (d, $J=11.81 \mathrm{~Hz}, \mathrm{HC}-11), 7.17(\mathrm{~d}, J=11.81 \mathrm{~Hz}$, $\mathrm{HC}-12$ ), 3.79 (s, $\left.\mathrm{CH}_{3} \mathrm{O}-1\right), 3.53$ (s, $\left.\mathrm{CH}_{3} \mathrm{O}-2\right), 3.85$ (s, $\mathrm{CH}_{3} \mathrm{O}-$ 3), 1.88 (s, $\left.\mathrm{H}_{3} \mathrm{C}-14\right), 8.65$ (d, J =7.36 Hz, NH). ${ }^{13} \mathrm{C}$ NMR (75 $\left.\mathrm{MHz}, \mathrm{DMSO}-d_{6}\right) \delta / \mathrm{ppm}$ : 150.03 (C-1), 125.74 (C-1a), 140.74 (C-2), 153.16 (C-3), 107.89 (C-4), 134.33 (C-4a), 29.27 (C-5), 36.96 (C-6), 51.73 (C-7), 150.21 (C-7a), 124.36 (C-8), 171.95 (C-9), 168.67 (C-10), 118.32 (C-11), 140.28 (C-12), 134.81 (C-12a), $60.84\left(\mathrm{CH}_{3} \mathrm{O}-1\right), 60.81\left(\mathrm{CH}_{3} \mathrm{O}-2\right)$, $55.73\left(\mathrm{CH}_{3} \mathrm{O}-3\right), 168.94$ (C-13), 22.52 (C-14). ${ }^{1} \mathrm{H}$ NMR $(300$ $\mathrm{MHz}, \mathrm{CD}_{3} \mathrm{CN}$ ) $\delta / \mathrm{ppm}: 6.73$ (s, HC-4). 2.21 (m, $\left.\mathrm{H}_{2} \mathrm{C}-5\right), 2.56$ (m, $\left.\mathrm{H}_{2} \mathrm{C}-5\right), 1.97\left(\mathrm{~m}, \mathrm{H}_{2} \mathrm{C}-6\right), 2.25\left(\mathrm{~m}, \mathrm{H}_{2} \mathrm{C}-6\right), 4.40$ (m, HC7), 7.47 (s, HC-8), 7.25 (d, J = $7.49 \mathrm{~Hz}, \mathrm{HC}-11), 7.49$ (d, J $=7.24 \mathrm{~Hz}, \mathrm{HC}-12$ ), 3.61 (s, $\left.\mathrm{CH}_{3} \mathrm{O}-1\right), 3.84$ (s, $\left.\mathrm{CH}_{3} \mathrm{O}-2\right), 3.87$ (s, $\left.\mathrm{CH}_{3} \mathrm{O}-3\right), 1.90$ (s, $\left.\mathrm{H}_{3} \mathrm{C}-14\right), 7.54$ (d, J =4.39 Hz, NH). ${ }^{13} \mathrm{C}$ NMR (75 MHz, $\mathrm{CD}_{3} \mathrm{CN}$ ) $\delta / p p m: 151.09$ (C-1), 126.50 (C-1a), 141.78 (C-2), 154.39 (C-3), 108.49 (C-4), 135.86 (C-4a), 30.11 (C-5), 37.93 (C-6), 53.41 (C-7), 150.32 (C-7a), 124.91 (C-8), 173.02 (C-9), 168.38 (C-10), 117.82 (C-11), 142.28 (C-12), 136.18 (C-12a), $61.59\left(\mathrm{CH}_{3} \mathrm{O}-1\right), 61.38\left(\mathrm{CH}_{3} \mathrm{O}-2\right)$, $56.56\left(\mathrm{CH}_{3} \mathrm{O}-3\right), 170.46$ (C-13), 22.71 (C-14). UV-visible in $\mathrm{CH}_{3} \mathrm{OH} \lambda_{\max } / \mathrm{nm}\left(\varepsilon / \mathrm{mol}^{-1} \mathrm{dm}^{3} \mathrm{~cm}^{-1}\right): 349$ (16917), 231 (28146).

\section{(8) Complex Colchiceine - Potassium Thiocyanate} 109 mg; yield $91 \%$; m.p. $=98-100{ }^{\circ} \mathrm{C}$, Anal. Calcd. for $\mathrm{C}_{21} \mathrm{H}_{23} \mathrm{NO}_{6} \cdot \mathrm{KSCN} \cdot 2.5 \mathrm{H}_{2} \mathrm{O}: \mathrm{C}, 50.09 ; \mathrm{H}, 5.31 ; \mathrm{N}, 5.31$. Found: C, 49.98; H, 5.36; N, 5.29. IR (KBr) v//cm-1: $3360 \mathrm{~m}, 3256 \mathrm{~m}$, 3052 w, 2999 w, 2938 w, 2856 w, 2837 w, 1661 s, 1609 s, 1544 m, 1489 m, 1453 m, 1274 m, 1196 m, 1136 m, 2051 $\mathrm{m}$. IR (nujol/fluorolub) $\tilde{v} / \mathrm{cm}^{-1}: 3370 \mathrm{~m}, 3249 \mathrm{~m}, 3052 \mathrm{w}$, 2952 w, 2936 w, 2856 w, 2727 w, 1658 s, 1608 s, 1539 m, 1488 m, 1456 m, 1404 m, 1348 m, 1274 m, 2054 s. ${ }^{1 H}$ NMR (300 MHz, DMSO- $d_{6}$ ) $\delta / p p m: ~ 6.82$ (s, HC-4), 2.14 (m, $\mathrm{H}_{2} \mathrm{C}-$ 5), $2.53\left(\mathrm{~m}, \mathrm{H}_{2} \mathrm{C}-5\right), 1.96\left(\mathrm{~m}, \mathrm{H}_{2} \mathrm{C}-6\right), 2.11\left(\mathrm{~m}, \mathrm{H}_{2} \mathrm{C}-6\right), 4.36$ (m, HC-7), 7.33 (s, HC-8), 7.36 (d, J=11.81 Hz, HC-11), 7.18 (d, $J=11.81 \mathrm{~Hz}, \mathrm{HC}-12), 3.79$ (s, $\left.\mathrm{CH}_{3} \mathrm{O}-1\right), 3.53\left(\mathrm{~s}, \mathrm{CH}_{3} \mathrm{O}-2\right)$, 3.85 (s, $\left.\mathrm{CH}_{3} \mathrm{O}-3\right), 1.88$ (s, H3C-14), 8.67 (d, J = $7.42 \mathrm{~Hz}, \mathrm{NH}$ ). ${ }^{13} \mathrm{C}$ NMR (75 MHz, DMSO- $d_{6}$ ) $\delta / p p m: 150.04$ (C-1), 125.73 (C-1a), 140.72 (C-2), 153.16 (C-3), 107.89 (C-4), 134.36 (C4a), 29.26 (C-5), 36.95 (C-6), 51.73 (C-7), 150.24 (C-7a), 124.38 (C-8), 171.92 (C-9), 168.63 (C-10), 118.32 (C-11), 140.29 (C-12), 134.80 (C-12a), $60.84\left(\mathrm{CH}_{3} \mathrm{O}-1\right), 60.81\left(\mathrm{CH}_{3} \mathrm{O}-\right.$ 2), $55.95\left(\mathrm{CH}_{3} \mathrm{O}-3\right), 168.93$ (C-13), 22.51 (C-14), 129.78 (SCN). ${ }^{1 H}$ NMR (300 MHz, $\mathrm{CD}_{3} \mathrm{CN}$ ) $\delta / p p m: 6.73$ (s, HC-4), $2.22\left(m, \mathrm{H}_{2} \mathrm{C}-5\right), 2.54\left(\mathrm{~m}, \mathrm{H}_{2} \mathrm{C}-5\right), 1.99\left(\mathrm{~m}, \mathrm{H}_{2} \mathrm{C}-6\right), 2.24$ (m, $\mathrm{H}_{2} \mathrm{C}-6$ ), 4.39 (m, HC-7), 7.48 (s, HC-8), 7.23 (d, J= $7.48 \mathrm{~Hz}$, $\mathrm{HC}-11$ ), 7.48 (d, J=7.23 Hz, HC-12), 3.61 (s, $\left.\mathrm{CH}_{3} \mathrm{O}-1\right), 3.82$ (s, $\mathrm{CH}_{3} \mathrm{O}-2$ ), 3.88 (s, $\left.\mathrm{CH}_{3} \mathrm{O}-3\right), 1.92$ (s, $\left.\mathrm{H}_{3} \mathrm{C}-14\right), 7.44$ (d, J = 4.39 $\mathrm{Hz}, \mathrm{NH}) .{ }^{13} \mathrm{C}$ NMR $\left(75 \mathrm{MHz}, \mathrm{CD}_{3} \mathrm{CN}\right.$ ) $\delta / \mathrm{ppm}: 151.15$ (C-1), 126.59 (C-1a), 141.81 (C-2), 154.37 (C-3), 108.48 (C-4), 135.85 (C-4a), 30.14 (C-5), 37.92 (C-6), 53.36 (C-7), 151.36 (C-7a), 124.83 (C-8), 173.03 (C-9), 168.49 (C-10), 117.71 (C11), 142.16 (C-12), 136.10 (C-12a), $61.55\left(\mathrm{CH}_{3} \mathrm{O}-1\right), 61.34$ $\left(\mathrm{CH}_{3} \mathrm{O}-2\right), 56.54\left(\mathrm{CH}_{3} \mathrm{O}-3\right), 170.69$ (C-13), 22.70 (C-14), 131.49 (SCN). UV-visible in $\mathrm{CH}_{3} \mathrm{OH} \lambda_{\max } / \mathrm{nm}\left(\varepsilon / \mathrm{mol}^{-1} \mathrm{dm}^{3}\right.$ $\mathrm{cm}^{-1}$ ): 350 (16200), 230 (28341).

(2) Colchiceine $\mathrm{C}_{21} \mathrm{H}_{23} \mathrm{NO}_{6} \cdot \mathrm{H}_{2} \mathrm{O}$, m.p. $148-150{ }^{\circ} \mathrm{C}$.

\section{Physical Measurements}

The NMR spectra of $\mathbf{2}$ and its 1:1 complexes $\mathbf{3}-\mathbf{8}\left(0.07 \mathrm{~mol} \mathrm{~L}^{-1}\right)$ with monovalent metal cations salts were recorded in $\mathrm{CD}_{3} \mathrm{CN}$ solutions using a Varian Gemini $300 \mathrm{MHz}$ spectrometer. All spectra were locked to deuterium resonance of $\mathrm{CD}_{3} \mathrm{CN}$. The ${ }^{1} \mathrm{H}$ NMR measurements in $\mathrm{CD}_{3} \mathrm{CN}$ were carried out at the operating frequency $300.075 \mathrm{MHz}$; flip angle $45^{\circ}$; spectral width $4500 \mathrm{~Hz}$; acquisition time $2.0 \mathrm{~s}$; relaxation delay $1.0 \mathrm{~s}$ and $T=293.0 \mathrm{~K}$, using TMS as the internal standard. No window function or zero filling was used. Digital resolution was $0.2 \mathrm{~Hz}$ per point. The error of chemical shift value was $0.01 \mathrm{ppm} .{ }^{13} \mathrm{C}$ NMR spectra were recorded at the operating frequency $75.454 \mathrm{MHz} ; \mathrm{pw}=60^{\circ} ; \mathrm{sw}=19000 \mathrm{~Hz}$; at $=1.8 \mathrm{~s} ; d_{1}$ $=1.0 \mathrm{~s} ; T=293.0 \mathrm{~K}$ and TMS as the internal standard. Line broadening parameters were 0.5 or $1 \mathrm{~Hz}$. The error of chemical shift value was $0.01 \mathrm{ppm}$. The ${ }^{1 \mathrm{H}}$ and ${ }^{13} \mathrm{C}$ NMR signals were assigned for each species using one or twodimensional (COSY, HETCOR, HMBC in inverse mode) spectra. 
Table 1. Value of $\lambda_{\max }$ and $\varepsilon$ in UV-Vis spectra of 2 and its complexes $3-8$ in $\left(\mathrm{CH}_{3} \mathrm{OH}\right)$

\begin{tabular}{ccccc}
\hline Compound & $\lambda_{\max 1} / \mathrm{nm}$ & $\varepsilon_{1} /\left(\mathrm{mol}^{-1} \mathrm{dm}^{3} \mathrm{~cm}^{-1}\right)$ & $\lambda_{\max 2} / \mathrm{nm}$ & $\varepsilon_{2} /\left(\mathrm{mol}^{-1} \mathrm{dm}^{3} \mathrm{~cm}^{-1}\right)$ \\
\hline $\mathbf{2}$ & 350.5 & 34887 & 243.5 & 62699 \\
$\mathbf{3}$ & 349.5 & 17444 & 224 & 35853 \\
$\mathbf{4}$ & 350 & 16762 & 223 & 36109 \\
$\mathbf{5}$ & 350.5 & 17292 & 222.5 & 39813 \\
$\mathbf{6}$ & 348.5 & 16192 & 231 & 27017 \\
$\mathbf{7}$ & 349 & 16917 & 231 & 28146 \\
$\mathbf{8}$ & 350 & 16200 & 230 & 28341 \\
\hline
\end{tabular}

The FT-IR spectra of 2 and its 1:1 complex 3-8 (0.07 $\mathrm{mol} \mathrm{dm}-3$ ) were recorded in the mid infrared region in $\mathrm{KBr}$ pellets, fluorolub/ nujol mixture for obtained complexes 38 and in $\mathrm{CD}_{3} \mathrm{CN}$ for colchiceine and its complex 6 with $\mathrm{LiClO}_{4}$. The spectra were taken with an IFS $113 \mathrm{v}$ FT-IR spectrophotometer (Bruker, Karlsruhe) equipped with a DTGS detector; resolution $2 \mathrm{~cm}^{-1}$, NSS=125. A cell with $\mathrm{Si}$ windows and wedge-shaped layers was used to avoid interferences (mean layer thickness $170 \mu \mathrm{m}$ ). Each FT-IR spectrum was measured by acquisition of 64 scans. All manipulations with the substances were performed in a carefully dried and $\mathrm{CO}_{2}$-free glove box.

The UV-Vis spectra were recorded in methanol by JASCO V-550 spectrophotometer at 200-600 nm range.

Liquid secondary ion mass spectra (FAB MS) were obtained with AMD 604 two sector mass spectrometer of reverse $B / E$ geometry, made by AMD Intectra (Germany). A $\mathrm{Csl}$ gun supplied the primary ion beam $\left(12 \mathrm{keV}, \mathrm{Cs}^{+}\right)$. The secondary ion beam was accelerated to $8 \mathrm{kV}$. Samples of the complexes studied were dissolved in 3-nitrobenzyl alcohol (Aldrich).

Elemental analysis of colchiceine complexes was carried out by means of a Elementarz Analyser Vario EL III. Melting point was determined on BUCHI SMP-20. MeltTemp II apparatus (Laboratory Devices Inc.)

\section{RESULTS AND DISCUSSION}

Colchiceine and respective inorganic salts $\left(\mathrm{LiClO}_{4}\right.$, Lil, $\mathrm{NaClO}_{4}, \mathrm{Nal}, \mathrm{KSCN}, \mathrm{KI}$ ) at the ratio 1:1 $\mathrm{M}$ were dissolved in methanol and were stirred for $24 \mathrm{~h}$ at room temperature. Compounds 3-8 were obtained as pale to dark yellow solids with very good yields. The complexes were studied by spectral analysis: UV-Vis, ${ }^{1} \mathrm{H}$ and ${ }^{13} \mathrm{C}$ NMR, FT-IR and MS FAB.

\section{UV-Vis Measurements}

The UV-Vis spectrum of colchiceine $\mathbf{2}$ in methanol (Table 1.) shows two bands at $\lambda_{\max 1} 350$ and $\lambda_{\max 2} 243.5 \mathrm{~nm}$ assigned to the tropolonic and benzenoic moieties in the colchiceine molecule, respectively. The values of $\varepsilon_{1}$ and $\varepsilon_{2}$ change much in comparison to colchiceine but those of $\varepsilon_{2}$ are higher for the colchiceine complexes with iodides 3-5 and lower for the complexes with perchlorate salts 6-7 and thiocyanate 8. The UV-Vis data for the 1:1 complexes of colchicine with monovalent cations 3-8 in methanol show the influence of salts only on the $\lambda_{\max 2}$ and $\varepsilon_{2}$ values, which means that the effect of coordination process is stronger on benzenoic moiety than on tropolonic moiety. In the UV-Vis spectra of the benzenoic moiety, hypsochromic shifts were observed for all obtained colchiceine complexes 3-8 of both $\lambda_{\max 1}$ and $\lambda_{\max 2}$ for iodide and perchlorate salts. The hypsochromic shifts of $\lambda_{\max 1}$ for tropolonic moiety is very weak. The hypsochromic shift of $\lambda_{\max 2}$ observed for colchiceine complexes with iodide salts $\mathbf{3}-\mathbf{5}$ was stronger than that for perchlorates 6-7 and thiocyanate $\mathbf{8}$, and it was the strongest for the complex of colchiceine with potassium iodide 5 , which means that the type of anion also influences the coordination process.

\section{NMR Measurements}

The NMR spectra of colchiceine complexes 3-8 were recordered in two different solvents to compare a possible interaction between the ligand (colchiceine) and the solvent as well as to establish the effect of the nature of solvents on the values of chemical shifts. The ${ }^{1} \mathrm{H}$ and ${ }^{13} \mathrm{C}$ NMR data of $\mathbf{2}$ and its complexes with iodides 3-5, perchlorates 6-7 and thiocyanate 8 in DMSO- $d_{6}$ and in $\mathrm{CD}_{3} \mathrm{CN}$ solution are given in Experimental Section.

\section{${ }^{1} \mathrm{H}$ NMR Spectra}

The chemical shift of proton at $\mathrm{OH}$ (hydroxyl group at C-10) in $\mathbf{2}$ cannot be obtained experimentally on account of the very low activation energy. Colchiceine exists in the form of two tautomers, which follows from intramolecular proton transfer on the tropolone moiety (between two oxygens at C-9 and C-10). ${ }^{[27]}$

The ${ }^{1} \mathrm{H}$ NMR spectra of colchiceine complexes 3-8 do not show significant changes in the values of chemical shifts of respective hydrogen atoms, as has been also observed earlier for other biologically active compounds like monensin, oligomycin and complexes of colchicine. ${ }^{[25,28-32]}$

The slightest changes of proton chemical shifts have been observed for the amide group. Apart from these values, ${ }^{1} \mathrm{H}$ NMR spectra give no additional information on the structure of the complexes studied. 
Table 2. Selected chemical shifts of protons in the ${ }^{1} \mathrm{H} N M R$ in $\mathrm{CD}_{3} \mathrm{CN}$

\begin{tabular}{|c|c|c|c|c|c|c|c|}
\hline \multirow{2}{*}{$\begin{array}{c}\text { Hydrogen } \\
\text { atom }\end{array}$} & \multicolumn{7}{|c|}{ Chemical shifts (ppm), $\delta_{\mathrm{H}}$ multiplicity, J / Hz } \\
\hline & 2 & 3 & 4 & 5 & 6 & 7 & 8 \\
\hline \multirow[t]{2}{*}{$\mathrm{H}_{2} \mathrm{C}-5$} & $2.18 \mathrm{~m}$ & $2.15 \mathrm{~m}$ & $2.21 \mathrm{~m}$ & $2.21 \mathrm{~m}$ & $2.22 \mathrm{~m}$ & $2.21 \mathrm{~m}$ & $2.22 \mathrm{~m}$ \\
\hline & $2.54 \mathrm{~m}$ & $2.55 \mathrm{~m}$ & $2.54 \mathrm{~m}$ & $2.55 \mathrm{~m}$ & $2.56 \mathrm{~m}$ & $2.56 \mathrm{~m}$ & $2.54 \mathrm{~m}$ \\
\hline \multirow[t]{2}{*}{$\mathrm{H}_{2} \mathrm{C}-6$} & $2.09 \mathrm{~m}$ & $2.09 \mathrm{~m}$ & $2.08 \mathrm{~m}$ & $2.03 \mathrm{~m}$ & $1.96 \mathrm{~m}$ & $1.97 \mathrm{~m}$ & $1.99 \mathrm{~m}$ \\
\hline & $2.23 \mathrm{~m}$ & $2.25 \mathrm{~m}$ & $2.25 \mathrm{~m}$ & $2.19 \mathrm{~m}$ & $2.26 \mathrm{~m}$ & $2.25 \mathrm{~m}$ & $2.24 \mathrm{~m}$ \\
\hline $\mathrm{HC}-8$ & $7.48 \mathrm{~s}$ & $7.51 \mathrm{~s}$ & $7.53 \mathrm{~s}$ & $7.52 \mathrm{~s}$ & $7.46 \mathrm{~s}$ & $7.47 \mathrm{~s}$ & $7.48 \mathrm{~s}$ \\
\hline HC-11 & 7.24 d 7.48 & 7.25 d 7.48 & $7.24 \mathrm{~d} 7.48$ & $7.25 \mathrm{~d} 7.48$ & $7.26 \mathrm{~d} 7.48$ & 7.25 d 7.49 & 7.23 d 7.48 \\
\hline $\mathrm{HN}$ & $7.16 \mathrm{~d} 6.32$ & $7.65 d 4.41$ & 7.54 d 4.42 & $7.44 \mathrm{~d} 4.42$ & $7.48 \mathrm{~d} 4.37$ & 7.54 d 4.39 & 7.44 d 4.39 \\
\hline
\end{tabular}

The ${ }^{1} \mathrm{H}$ NMR spectra of colchiceine complexes $3-8$ in acetonitrile, similarly as those of colchiceine complexes 38 in DMSO- $d_{6}$, show the signals of protons assigned to three o-methoxyl groups whose positions do not change much after the complexation process. Some selected data on proton chemical shifts in acetonitrile are given in the Table 2. Slight changes in chemical shifts are observed for methylene protons at carbon atoms $\mathrm{C} 5$ and $\mathrm{C} 6$ (ring B). The proton at the carbon atom $\mathrm{C}-8$ observed as a singlet showed considerable change in chemical shift for colchiceine complex with sodium perchlorate 4 .

The differences in chemical shifts of protons of the acetamide substituent at C-7 position gave the greatest changes, as a result of the complexation process, changing from $7.16 \mathrm{ppm}$ in $\mathbf{2}$ to $\mathbf{7 . 4 4}-\mathbf{7 . 6 5} \mathrm{ppm}$ in $\mathbf{3} \mathbf{- 8}$. The proton signal whose position was the most changed in this region of the spectra of complexes 3-8 was observed in colchiceine complex with lithium perchlorate 6 . The changes in the values of chemical shift decrease in the following order $\mathrm{Li}^{+}>\mathrm{Na}^{+}>\mathrm{K}^{+}$, which suggests that the larger the cation, the weaker the complex interaction with the acetamide group. Figure 2 . shows the changes in the position of the chemical shift of proton at $\mathrm{NH}$ group of acetamide substituent in colchiceine and its complexes with lithium iodide $\mathbf{3}$ and perchlorate $\mathbf{6}$.

If only lithium cation had impact on the complexation process in both complexes $\mathbf{3}$ and $\mathbf{6}$, the position of the chemical shift of proton from the $\mathrm{NH}$ group would be almost the same, Figure 2. It can be easily observed that the type of anion also influences the position of proton in the acetamide group.

\section{${ }^{13}$ C NMR spectra}

Some selected data on chemical shifts in ${ }^{13} \mathrm{C}$ NMR spectra of colchiceine complexes 3-8 in DMSO- $d_{6}$ are given in the Table $3 .{ }^{13} \mathrm{C}$ NMR spectra of these complexes are much more informative than ${ }^{1} \mathrm{H}$ NMR spectra. The chemical shifts of $o$-methoxy carbons do not change much after the complexation process and the most shifted ones are those in complex $\mathbf{7}$ and $\mathbf{8}$. The differences in chemical shifts of

Table 3. Selected carbon-13 chemical shifts for colchiceine 2 and its complexes 3-8 (in DMSO- $d_{6}$ )

\begin{tabular}{|c|c|c|c|c|c|c|c|}
\hline \multirow[t]{2}{*}{ Carbon atom } & \multicolumn{7}{|c|}{ Chemical shifts $(\delta / p p m)$} \\
\hline & 2 & 3 & 4 & 5 & 6 & 7 & 8 \\
\hline C-1 & 149.73 & 149.68 & 149.75 & 149.75 & 149.82 & 150.03 & 150.04 \\
\hline $\mathrm{C}-1 \mathrm{a}$ & 125.53 & 125.60 & 125.56 & 125.49 & 125.59 & 125.74 & 125.73 \\
\hline $\mathrm{C}-2$ & 140.49 & 140.48 & 140.50 & 140.46 & 140.55 & 140.74 & 140.72 \\
\hline $\mathrm{C}-3$ & 152.87 & 152.82 & 152.88 & 152.85 & 152.95 & 153.16 & 153.16 \\
\hline C-4a & 134.05 & 133.69 & 133.98 & 134.07 & 134.14 & 134.33 & 134.36 \\
\hline C-7 & 51.58 & 51.61 & 51.65 & 51.62 & 51.69 & 51.73 & 51.73 \\
\hline$C-7 a$ & 149.95 & 149.94 & 149.95 & 149.91 & 150.02 & 150.21 & 150.24 \\
\hline $\mathrm{C}-8$ & 124.06 & 123.91 & 124.07 & 124.07 & 124.18 & 124.36 & 124.38 \\
\hline$C-9$ & 171.57 & 172.00 & 171.74 & 171.57 & 171.71 & 171.95 & 171.92 \\
\hline$C-10$ & 168.35 & 168.54 & 168.53 & 168.32 & 16.47 & 168.67 & 168.63 \\
\hline$C-11$ & 118.07 & 118.07 & 118.12 & 118.08 & 118.16 & 118.32 & 118.32 \\
\hline$C-12$ & 139.96 & 139.91 & 140.00 & 139.99 & 140.09 & 140.28 & 140.29 \\
\hline$C-12 a$ & 134.52 & 134.54 & 134.56 & 134.52 & 134.63 & 134.81 & 134.80 \\
\hline $\mathrm{CH}_{3} \mathrm{O}-1$ & 60.70 & 60.71 & 60.74 & 60.70 & 60.80 & 60.84 & 60.84 \\
\hline $\mathrm{CH}_{3} \mathrm{O}-2$ & 60.66 & 60.69 & 60.72 & 60.67 & 60.77 & 60.81 & 60.81 \\
\hline $\mathrm{CH}_{3} \mathrm{O}-3$ & 55.82 & 55.87 & 55.91 & 55.88 & 55.91 & 55.73 & 55.95 \\
\hline$C-13$ & 168.49 & 168.90 & 168.61 & 168.53 & 168.69 & 168.94 & 168.93 \\
\hline
\end{tabular}


a)

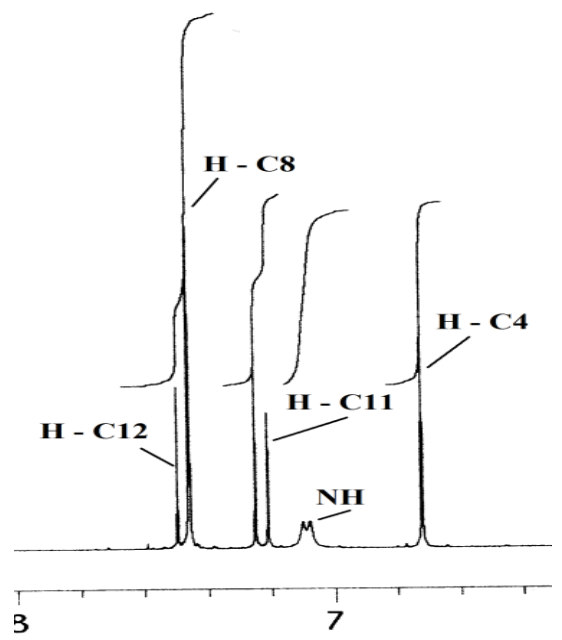

b)

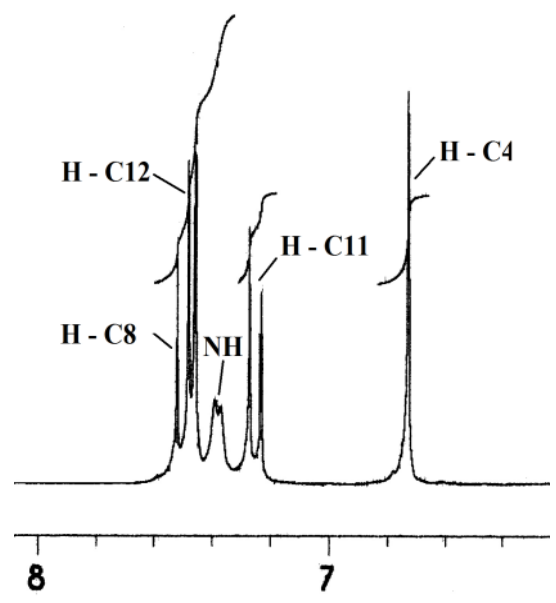

c)

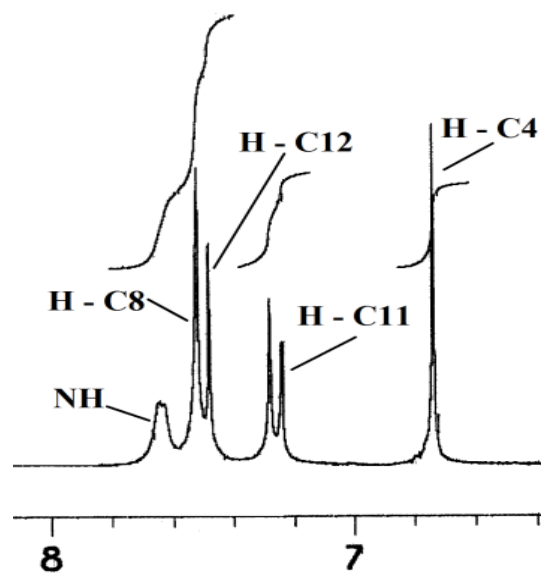

Figure 2. ${ }^{1} \mathrm{H}$ NMR spectra $\left(\mathrm{CD}_{3} \mathrm{CN}\right)$ in the range of $6.4-8.2$ ppm of: a) colchiceine 2, b) complex with lithium perchlorate 6 and c) complex with lithium iodide 3 . carbon atom $\mathrm{C}-13$ of carbonyl group do not depend on the type of monovalent cation. Signal assigned to carbonyl carbon atom C-13 is most shifted in 3, $\mathbf{6}$ and $\mathbf{7}$ ranging from 168.90 to 168.95 ppm (168.49 ppm in 2). Signal of carbonyl carbon atom C-9 for colchiceine appears at $171.57 \mathrm{ppm}$, but for colchiceine complexes with lithium iodide 3 shifts to 172 ppm. The chemical shifts of carbonyl carbon atom C-9 decrease in the following order $\mathrm{Li}^{+} \rightarrow \mathrm{Na}^{+} \rightarrow \mathrm{K}^{+}$for iodides and $\mathrm{Na}^{+} \rightarrow \mathrm{K}^{+} \rightarrow \mathrm{Li}^{+}$for perchlorates $\mathbf{6}$ and $\mathbf{7}$ and thiocyanate 8. This observation suggests that in complexation process in colchiceine complex with lithium iodide $\mathbf{3}$ both carbonyl groups are involved. Some changes after complexation process are also visible in chemical shifts for carbon atoms on ring $\mathrm{B}$. For carbon atoms $\mathrm{C}-7$ and $\mathrm{C}-7 \mathrm{a}$ the greatest change in chemical shifts are observed in colchiceine complex 7 and 8. The carbon atoms C-11, C- 12 and C-12a on the $\mathrm{C}$ ring shift much in colchiceine complexes $\mathbf{7}$ and $\mathbf{8}$. For carbon atoms on ring A C-1, C- 1 a, C-2, C-3, C-4a the greatest change in chemical shifts are observed in colchiceine complex $\mathbf{7}$ and $\mathbf{8}$.

Some selected ${ }^{13} \mathrm{C}$ NMR chemical shifts of colchiceine complexes 3-8 are given in the Table 4. The ${ }^{13} \mathrm{C}$ NMR spectra of colchiceine complexes 3-8 in $\mathrm{CD}_{3} \mathrm{CN}$ similarly to those of complexes 3-8 in DMSO- $d_{6}$, show the signals of carbons assigned to three $o$-methoxyl groups do not change much after the complexation process. The chemical shifts of carbon atoms from the three $o$-methoxyl groups decrease in the following order $\mathrm{Li}^{+} \rightarrow \mathrm{Na}^{+} \rightarrow \mathrm{K}^{+}$, both for iodides and perchlorates.

The chemical shifts of carbon atoms show that both carbonyl group at C-7 position (acetamide group) and C-9 in the tropolone ring $C$ are involved in the coordination. The complexation process of $\mathrm{Li}^{+}, \mathrm{Na}^{+}$and $\mathrm{K}^{+}$cations results in changes in the chemical shifts of the carbon atoms of the tropolone ring $\mathrm{C}$ of colchiceine and at the acetamide substituent (carbon from carbonyl group) at C-7 position. The chemical shifts of carbon atom C-13 of acetamide group depend on the type of monovalent cation and increase in the following order $\mathrm{Li}^{+} \rightarrow \mathrm{Na}^{+} \rightarrow \mathrm{K}^{+}$for iodides $3-5$ and $\mathrm{K}^{+} \rightarrow$ $\mathrm{Li}^{+} \rightarrow \mathrm{Na}^{+}$for perchlorates 6-7 and thiocyanate 8 . The most upfield shift of C-13 carbonyl carbon atom was observed in 6 (complex with lithium perchlorate), while the most downfield shift was found in $\mathbf{8}$ with respect to $\mathbf{2}$. On the other hand signal of carbonyl carbon atom C-9 in colchiceine appears at $173.30 \mathrm{ppm}$, but for colchiceine complexes with lithium iodide 3 shifts slightly downfield to $172.94 \mathrm{ppm}$. The chemical shifts of carbonyl carbon atom C-9 decrease in the following order $\mathrm{Li}^{+} \rightarrow \mathrm{Na}^{+} \rightarrow \mathrm{K}^{+}$for iodides and for perchlorates $\mathbf{6}$ and $\mathbf{7}$ and thiocyanate 8 .

\section{FT-IR Measurements}

To be able to make comparison to the results of the previous work on colchicine complexes, ${ }^{[25]}$ the IR spectra of 
Table 4. Selected chemical shifts of carbon atoms in the ${ }^{13} \mathrm{CNMR}$ in $\mathrm{CD}_{3} \mathrm{CN}$

\begin{tabular}{cccccccc}
\hline \multirow{2}{*}{ Carbon atom } & \multicolumn{7}{c}{ Chemical shifts $(\mathrm{ppm}) \mathrm{CD}_{3} \mathrm{CN}$} \\
\hline $\mathrm{C}-1$ & $\mathbf{3}$ & $\mathbf{4}$ & $\mathbf{5}$ & $\mathbf{6}$ & $\mathbf{7}$ & $\mathbf{8}$ \\
\hline $\mathrm{C}-1 \mathrm{a}$ & 151.44 & 151.04 & 150.99 & 151.00 & 151.08 & 151.09 & 151.15 \\
$\mathrm{C}-2$ & 126.83 & 126.37 & 126.46 & 126.48 & 126.41 & 126.50 & 126.59 \\
$\mathrm{C}-3$ & 142.15 & 141.74 & 141.67 & 141.70 & 141.78 & 141.78 & 141.81 \\
$\mathrm{C}-4 \mathrm{a}$ & 154.71 & 154.39 & 154.26 & 154.30 & 154.45 & 154.39 & 154.37 \\
$\mathrm{C}-5$ & 136.29 & 135.75 & 135.78 & 135.78 & 135.81 & 135.86 & 135.85 \\
$\mathrm{C}-7$ & 30.21 & 30.03 & 30.05 & 30.06 & 30.06 & 30.11 & 30.14 \\
$\mathrm{C}-7 \mathrm{a}$ & 53.47 & 53.53 & 53.37 & 53.35 & 53.49 & 53.41 & 53.36 \\
$\mathrm{C}-9$ & 151.56 & 151.31 & 151.30 & 151.29 & 151.19 & 150.32 & 151.36 \\
$\mathrm{C}-10$ & 173.30 & 172.94 & 173.06 & 173.09 & 172.98 & 173.02 & 173.03 \\
$\mathrm{C}-11$ & 168.74 & 168.43 & 168.41 & 168.24 & 168.48 & 168.38 & 168.49 \\
$\mathrm{C}-12$ & 117.81 & 118.20 & 117.88 & 117.66 & 117.95 & 117.82 & 117.71 \\
$\mathrm{C}-12 \mathrm{a}$ & 142.43 & 142.30 & 142.13 & 142.12 & 142.36 & 142.28 & 142.16 \\
$\mathrm{CH}{ }_{3} \mathrm{O}-1$ & 136.07 & 136.24 & 135.95 & 135.94 & 136.29 & 136.18 & 136.10 \\
$\mathrm{CH}{ }_{3} \mathrm{O}-2$ & 61.64 & 61.62 & 61.53 & 61.49 & 61.65 & 61.59 & 61.55 \\
$\mathrm{CH}_{3} \mathrm{O}-3$ & 61.46 & 61.30 & 61.30 & 61.28 & 61.35 & 61.38 & 61.34 \\
$\mathrm{C}-13$ & 56.66 & 56.53 & 56.50 & 56.49 & 56.54 & 56.56 & 56.54 \\
\hline
\end{tabular}

colchiceine and its complexes 3-8 were collected both in solid state $-\mathrm{KBr}$ pellets and in a nujol/fluorolub while those of $\mathbf{2}$ and $\mathbf{6}$ in $\mathrm{CD}_{3} \mathrm{CN}$ solutions. Initial examination of these spectra showed that some differences in position, shape and intensity of respective bands can be observed in spectra obtained in $\mathrm{KBr}$ pellets, nujol/fluorolub and in $\mathrm{CD}_{3} \mathrm{CN}$ solutions.

The FT-IR spectra of colchiceine complexes 3-5 with monovalent cations and, for comparison, the spectrum of colchiceine $\mathbf{2}$ all in $\mathrm{KBr}$ pellets are shown in Figure 3 and Figure 1S (Supporting Information).

From crystallographic studies it is well known that colchiceine in the solid state exist as a monohydrate. In the FT-IR spectrum, the stretching vibration of amine group observed as a broad band with a maximum at $3357 \mathrm{~cm}^{-1}$. The same band is also observed in the spectra of colchiceine complexes with monovalent cations $\mathbf{3 - 8}$, but the maximum of the broad band is shifted toward higher wavenumbers depending on the type of cation in the following order $\mathrm{K} \rightarrow \mathrm{Na} \rightarrow \mathrm{Li}$ for iodides 3-5 and $\mathrm{K} \approx \mathrm{Na} \rightarrow \mathrm{Li}$ in perchlorates 6-7 and thiocyanate 8. This observation indicates that in the solid, not only the cation but also the type of anion has impact on the complexation process. The strongest shift of this band is observed in 3. Cation and anion together with water molecules are involved in the complexation process. In the region of stretching vibrations of two carbonyl groups of tropolone and the acetamide group, respectively, these two bands are slightly shifted from $1611 \mathrm{~cm}^{-1}$ and $1653 \mathrm{~cm}^{-1}$ in 2 to the region of $1602-$ $1610 \mathrm{~cm}^{-1}$ and $1662-1653 \mathrm{~cm}^{-1}$ in colchiceine complexes 38. The strongest shift of the band assigned to the carbonyl group of tropolone ring $\mathrm{C}$ is observed in colchiceine complex with lithium iodide 3 . The strongest shift of the band assigned to the acetamide group is observed in

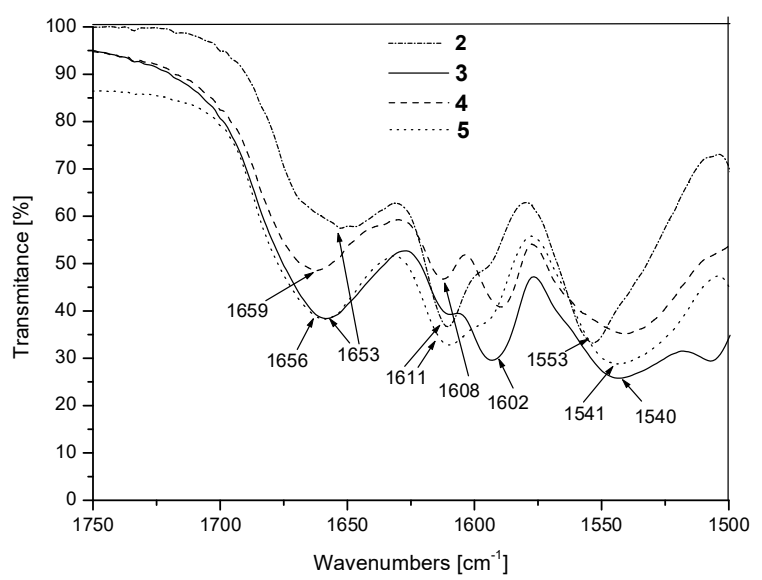

Figure 3. FT-IR spectra ( $\mathrm{KBr}$ ) of colchiceine 2 and its complexes with lithium iodide 3 , sodium iodide 4 and 


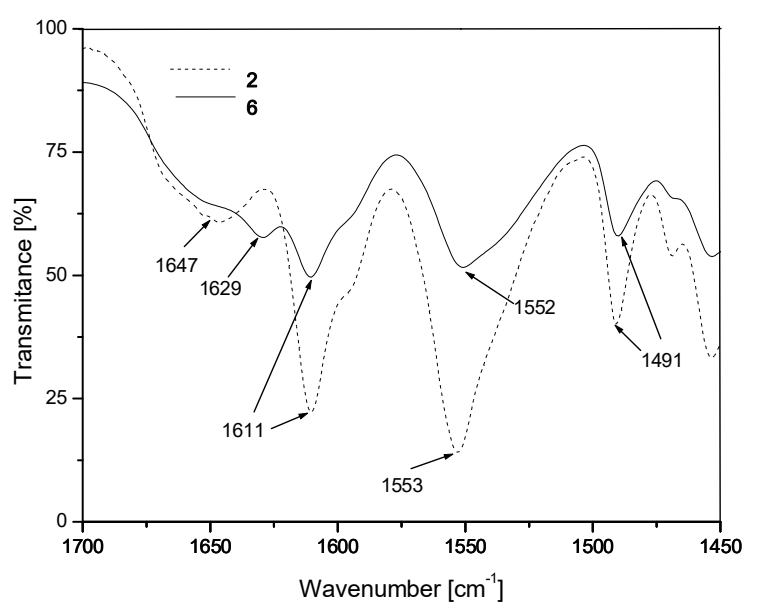

Figure 4. FT-IR spectra (nujol/fluorolub) of Colchiceine Complexes with lithium perchlorate 6 in the region of carbonyl group 1700-1450 $\mathrm{cm}^{-1}$.

colchiceine complex with sodium perchlorate 7 .

The FT-IR spectra of $\mathbf{1}$ show very characteristic absorption bands of tropolone ring in the colchicine molecule, appearing in the region of $1624-1605 \mathrm{~cm}^{-1}$, $1570-1538 \mathrm{~cm}^{-1}$ and $1280-1250 \mathrm{~cm}^{-1},{ }^{[26]}$ the first two are assigned to the stretching vibrations of $\tilde{v}_{(\mathrm{C}=\mathrm{C})}$, but in the IR spectra of colchiceine these bands appear in the region of $1553 \mathrm{~cm}^{-1}$ and $1276 \mathrm{~cm}^{-1}$. It was supposed that in the tropolone ring $\mathrm{C}$, the active hydrogen was statically located between the two oxygen atoms (on hydroxyl and carbonyl group) in such a way that the resonance was possible without zwitterion formation by shifts of the double bond and $\mathrm{OH}$ bonds as indicated. [33] The FT-IR spectra of colchiceine complexes 3-8 show the bands assigned to the stretching vibrations of amine group and hydroxyl group in the region of $3650-2500 \mathrm{~cm}^{-1}$. These bands are shifted towards higher wavenumbers in comparison to their position in the spectrum of $\mathbf{2}$, which is a result of the presence of water molecules in the crystalline structure of colchiceine complexes 3-8.

The FT-IR spectra in the nujol/fluorolub of respective complex 6 are shown in Figures 2S-3S (Supporting Information) and Figure 4, colchiceine is present as a monohydrate and in its FT-IR spectra the band assigned to water molecule is present. [34]

The stretching vibrations of this water molecule is observed in the FT-IR spectra as a band with a maximum at about $3447 \mathrm{~cm}^{-1}$. The same bands are also observed in the spectra of the colchiceine complexes with $\mathrm{Li}^{+}, \mathrm{Na}^{+}$and $\mathrm{K}^{+}$ cations, but their maximum is slightly shifted towards lower wavenumbers, in a way dependent on the type of cation in the following sequence: $\mathrm{K}^{+} \rightarrow \mathrm{Na}^{+} \rightarrow \mathrm{Li}^{+}$in iodides, which was also observed for the complexes of colchicine, ${ }^{[25]}$ and $\mathrm{Li}^{+} \rightarrow \mathrm{Na}^{+} \rightarrow \mathrm{K}^{+}$in perchlorates. In the FT-IR spectrum, the $\mathrm{NH}$ stretching vibrations of colchiceine produces the band with a maximum at $3359 \mathrm{~cm}^{-1}$. In the FT-IR spectra of colchiceine complexes the same band is observed but shifted towards lower wavenumbers, to $3245 \mathrm{~cm}^{-1}$ in complexes with iodide salts. The bands assigned to $\mathrm{NH}$ stretching vibrations of colchiceine complexes with iodides are much more shifted than those of the colchiceine complexes with perchlorates. The absence of any band in the usual carbonyl range $\left(1720-1670 \mathrm{~cm}^{-1}\right)$ can be explained by the conjunction of carbonyl group with unsaturated group or aromatic ring that lowers the carbonyl frequencies. In the region of stretching vibrations of $\mathrm{C}=\mathrm{O}$ group two bands are present, one assigned to the tropolone moiety and the other to acetamide groups. The shape and intensity of bands differ from those in the spectrum of colchiceine. The band of carbonyl group of tropolone moiety does not shifts much in comparison to the band assigned to the carbonyl group from acetamide, which shifts towards higher wavenumbers from $1647 \mathrm{~cm}^{-1}$ in 2 to $1655-1657 \mathrm{~cm}^{-1}$ in complexes 3-8 as a result of complexation process. Only in complex 6 this carbonyl band shifts toward lower wavenumbers from $1647 \mathrm{~cm}^{-1}$ to 1629 $\mathrm{cm}^{-1}$. The strongest shift of the bands assigned to $\tilde{v}_{(\mathrm{CO})}$ vibrations of acetamide group, from $1647 \mathrm{~cm}^{-1}$ in colchiceine to $1657 \mathrm{~cm}^{-1}$, was observed in its complex with $\mathrm{K}^{+} \mathbf{5}$, while for the tropolone carbonyl group the greatest shift was from $1611 \mathrm{~cm}^{-1}$ in 2 to $1602 \mathrm{~cm}^{-1}$ in complex 3 with lithium iodide.

The bands assigned to the tropolone moiety (unsaturated resonant system of ring C) appear at $1611 \mathrm{~cm}^{-1}, 1553 \mathrm{~cm}^{-1}, 1349 \mathrm{~cm}^{-1}$ and $1278 \mathrm{~cm}^{-1}$ in the FT-IR spectra of colchicine $\mathbf{1}$ and colchiceine $\mathbf{2}$. As expected, the same bands appear in the FT-IR spectra of colchiceine complexes 3-8 at somewhat different frequencies and with different relative intensities.

The FT-IR spectra of $\mathbf{2}$ and its complex with lithium perchlorate 6 in $\mathrm{CD}_{3} \mathrm{CN}$ solutions were also obtained and the data are given in Table 5 and shown in Figure 5 and Figure 4S (Supporting Information). For comparison, the data for colchicine $\mathbf{1}$ and its complex with lithium perchlorate 1a, are also given. ${ }^{[25]}$ Changes in the shape and intensity of the bands are easily observed. The bands assigned to the stretching vibrations of $\mathrm{NH}$ group are shifted to the lower wavenumbers from $3371 \mathrm{~cm}^{-1}$ for 2 to $3358 \mathrm{~cm}^{-1}$ for its complex 6 with $\mathrm{Li}^{+}$cation. In the region of stretching vibrations of $\mathrm{C}=\mathrm{O}$ bonds of the carbonyl group of tropolone and acetamide groups, two bands appear and shift towards lower wavenumbers in comparison to the FTIR spectrum of colchiceine. After the complexation process, the band assigned to the stretching vibrations of carbonyl group of acetamide group is shifted from $1681 \mathrm{~cm}^{-1}$ for 2 to $1662 \mathrm{~cm}^{-1}$ for 6 , which implies that the carbonyl group of 
Table 5. Wavenumbers $\left(\mathrm{cm}^{-1}\right)$ in FT-IR spectra in acetonitrile for 2 and its complex 6 and colchicine 1 and its complex with lithium perchlorate 1a. ${ }^{[25]}$

\begin{tabular}{ccccc}
\hline \multirow{2}{*}{ Assignments } & \multicolumn{4}{c}{ Wavenumbers $/ \mathrm{cm}^{-1}$} \\
& $\mathbf{2}$ & $\mathbf{6}$ & $\mathbf{1}$ & $\mathbf{1 a}$ \\
\hline$v \mathrm{OH}$ & 3538 & 3536 & 3544 & 3539 \\
$v \mathrm{NH}$ & 3371 & 3358 & 3369 & 3367 \\
& 3206 & 3229 & 3095 & 3163 \\
$v \mathrm{CH}$ & 3167 & 3160 & 3090 & 3002 \\
& 2998 & 2998 & 2943 & 2944 \\
$v \mathrm{C}=\mathrm{O}$ & 1681 & 1660 & 1680 & 1664 \\
$v \mathrm{C}=\mathrm{O}$ & 1617 & 1615 & 1618 & 1614 \\
$v \mathrm{C}=\mathrm{C}$ & 1596 & 1596 & 1591 & 1592 \\
$v \mathrm{C}=\mathrm{C}$ & 1556 & 1551 & 1575 & 1573 \\
\hline
\end{tabular}

acetamide substituent at C-7 rather is involved in the complexation process rather than the carbonyl group of tropolone ring $\mathrm{C}$. As can be concluded the complexation process involves especially the carbonyl group of acetamide substituent at C-7 rather than the carbonyl group of tropolone ring $\mathrm{C}$.

The presence of free perchlorate anions $\mathrm{ClO}_{4}{ }^{-}$is manifested by four fundamental vibration bands: symmetric stretching band at $\approx 932 \mathrm{~cm}^{-1}$, symmetric bending band at $\approx 460 \mathrm{~cm}^{-1}$, asymmetric stretching band at $\approx 1115 \mathrm{~cm}^{-1}$ and asymmetric bending band at $\approx 630$ $\mathrm{cm}^{-1} .{ }^{[35,36]}$ The IR spectra of colchiceine complexes: with lithium perchlorate $\mathbf{6}$ and with sodium perchlorate $\mathbf{7}$ show the bands assigned to perchlorate anions and their wavenumbers are given in the Table 6 and Figure 6. The broad band at $\approx 1115 \mathrm{~cm}^{-1}$ assigned to asymmetric stretching is shifted towards lower wavenumbers as a result of complexation process and also in case of presence of water molecules in complexes 6 and 7. These observations were made for the spectra of the samples in $\mathrm{KBr}$ pellet, nujol/fluorolub suspension and for complex $\mathbf{6}$ for $\mathrm{CD}_{3} \mathrm{CN}$ solution, which means that the medium in which the

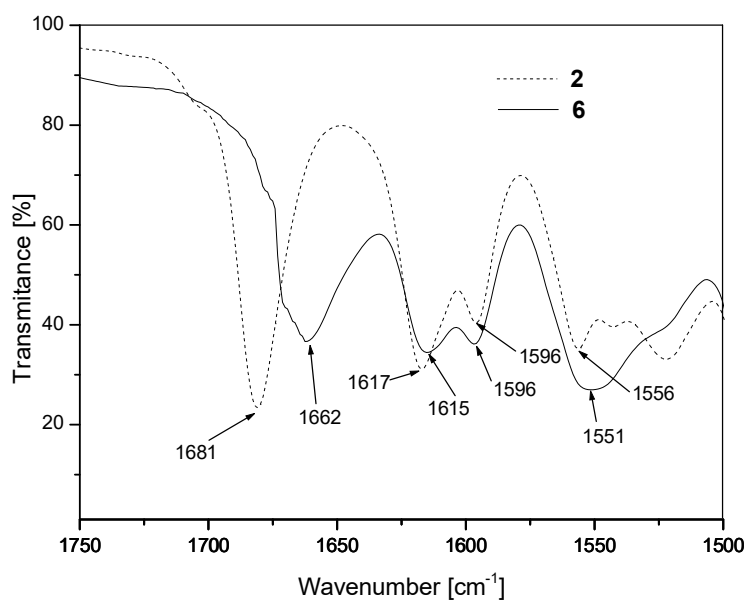

Figure 5. FT-IR spectra of Colchiceine 2 and its Complex with lithium perchlorate $6\left(\mathrm{CD}_{3} \mathrm{CN}\right): 1700-1575 \mathrm{~cm}^{-1}$.

IR spectra were recorded (solid state, suspension or solution) did not affect behavior of the band assigned to $\mathrm{ClO}_{4}{ }^{-}$group. The symmetric bending band usually present at $\approx 460 \mathrm{~cm}^{-1}$ after the complexation process is shifted towards higher wavenumbers for 6 and 7 to $484 \mathrm{~cm}^{-1}$, but these shifts are irrespective of the type of cation and the medium in which IR spectra were recorded (solid state, suspension or solution). The symmetric stretching band is shifted towards lower wavenumbers $918-920 \mathrm{~cm}^{-1}$ for the IR spectra in $\mathrm{KBr}$ and nujol/fluorolub and this change does not depend on type of cation, but in $\mathrm{CD}_{3} \mathrm{CN}$ solution for complex 6 only a slight change by $2 \mathrm{~cm}^{-1}$ is observed.

\section{FAB MS Measurements}

In Fast Atom Bombardment (FAB) MS mass spectra, molecular ions of colchiceine with respective monovalent metal ions as $\mathrm{Li}^{+}, \mathrm{Na}^{+}$and $\mathrm{K}^{+}$in stoichiometry 1:1 can be observed, respectively. The relevant data are given in the Table 7.

For all complexes, except 5, formation of complexes in stoichiometry in 2:1 is also observed. For complex 6

Table 6. Wavenumbers of free perchlorate anion and in complexed form of $\mathrm{ClO}_{4}^{-}$: as colchiceine complex with lithium perchlorate 6 and colchiceine complex with sodium perchlorate 7

\begin{tabular}{|c|c|c|c|c|c|c|}
\hline \multirow{3}{*}{ Assignment } & \multicolumn{6}{|c|}{ Wavenumbers $/ \mathrm{cm}^{-1}$} \\
\hline & \multirow[t]{2}{*}{ Free $\mathrm{ClO}_{4}^{-}$} & 6 & 6 & 6 & 7 & 7 \\
\hline & & $\mathrm{KBr}$ & nujol/fluorolub & $\mathrm{CD}_{3} \mathrm{CN}$ & $\mathrm{KBr}$ & nujol/fluorolub \\
\hline \multirow{2}{*}{ 1. Vas } & \multirow[b]{2}{*}{$\approx 1115$} & \multirow{2}{*}{$\begin{array}{l}1087 \\
1044\end{array}$} & \multirow{2}{*}{$\begin{array}{l}1094 \\
1011\end{array}$} & 1104 & 1108 & \multirow{2}{*}{$\begin{array}{l}1092 \\
1044\end{array}$} \\
\hline & & & & 1100 & 1090 & \\
\hline 2. $v_{\text {sym }}$ & $\approx 932$ & 918 & 920 & 930 & 918 & 919 \\
\hline 3. $\delta_{\mathrm{as}}$ & $\approx 630$ & 625 & 625 & 625 & 626 & 624 \\
\hline 4. $\delta_{\text {sym }}$ & $\approx 460$ & 484 & 483 & 483 & 484 & 484 \\
\hline
\end{tabular}




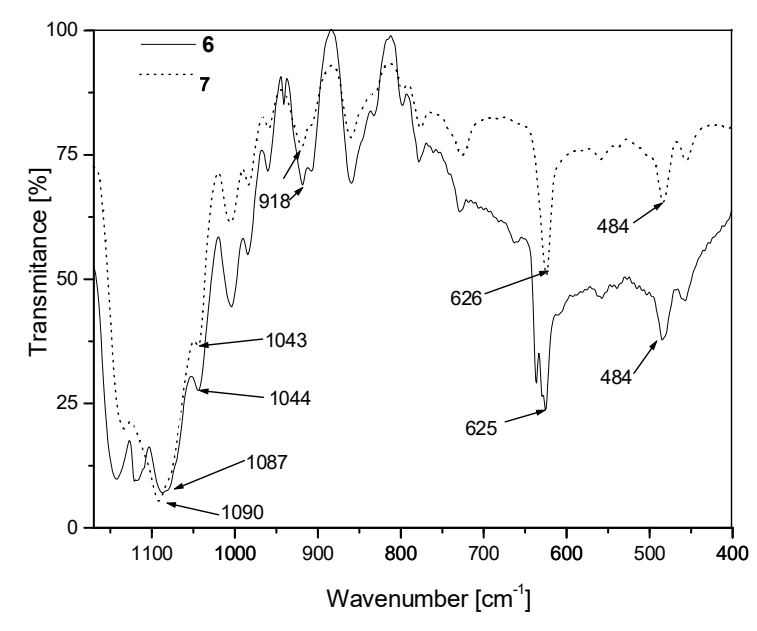

Figure 6. FT-IR spectra of colchiceine complex with lithium perchlorate 6 and colchiceine complex with sodium perchlorate 7 in the range of $1200-400 \mathrm{~cm}^{-1} \mathrm{ClO}_{4}{ }^{-}$anion.

signals at $m / z=392$ and also at $m / z=778$ appear, which indicates not only 1:1 but also 2:1 stoichiometry, of two colchiceine molecules and one lithium cation. Moreover, in 6 two other signals with low intensity (\%) were observed, which can be assigned to much more complicated stoichiometry with perchlorate anion at $m / z=498$, which indicates a connection colchiceine molecule to two lithium cations and one perchlorate anion and also $\mathrm{m} / \mathrm{z}=884$ two colchiceine molecules to two lithium cations and one perchlorate anion. It can be easily observed that formation of complicated stoichiometry depends on the kind of cation and decreases in the order lithium, sodium to potassium cation.

\section{CONCLUSIONS}

Series of new colchiceine complexes were obtained and described by spectral analysis. In the present work it was found that exchanging just one substituent in colchicine molecule at C-10 carbon atom from methoxyl to hydroxyl group on tropolone ring $\mathrm{C}$ causes huge changes in complexes formation. The strongest changes in the ${ }^{1} \mathrm{H}$ and ${ }^{13} \mathrm{C}$ NMR and in the FT-IR spectra were observed for colchiceine complexes with lithium iodide and lithium perchlorate.

In our previous work it has been found that colchicine forms complexes with lithium, sodium and potassium iodides, perchlorates and thiocyanate. In comparison to our previous work of colchicine complexes, colchiceine forms complexes in which in complexation process perchlorate anions are also involved, what was observed especially in colchiceine complex with lithium perchlorate.

Supplementary Information. Supporting information to the paper is enclosed to the electronic version of the article at: http://dx.doi.org/10.5562/cca2871.

\section{REFERENCES}

[1] A. Brossi, H. J. Yeh, M. Chrzanowska, J. Wolff, E. Hamel, C.M. Lin, F .Quin, M. Suffness, J. Silverton, Med. Res. Rev. 1988, 8, 77.

[2] M. Klintscher, C. Beham - Schmidt, H. Radner, G. Henning, P. Roll, Forensic Sci. Int. 1999, 106, 191.

[3] A. Körner, S. Kohn, J. Chromatogr. A. 2005, 1089, 141.

[4] M. Schonharting, G. Mende, G. Siebert, H.-S. Z. Physiol. Chem. 1974, 355, 1991.

[5] M. Cavazza, F. Pietra, Tetrahedron Lett. 2003, 44, 1895.

[6] M.P. Staretz, S.B. Hastie, J. Org. Chem. 1991, 56, 428.

[7] M. Cavazza, M. Zandomeneghi, F. Pietra, J. Chem. Soc. Perkin Trans. 1 2002, 560.

[8] G. Forni, G. Massarani, J. Chromatogr. 1977, 131, 444.

[9] A. E. Klein, P.J. Davis, Anal. Chem. 1980, 52, 2432.

[10] Y. H. Caplan, K. G. Orloff, B. C. Thompson, J. Anal. Toxicol. 1980, 4, 153.

[11] D. Jarvie, J. Park, M. J. Stewart, Clin. Tox. 1979, 14, 375.

[12] E. Lacey, R. L. Brady, J. Chromatogr. 1984, 315, 233.

[13] A. Maru`ska, O. Korny`sova, J. Chromatogr. A 2006, $1112,319$.

[14] T. Tateischi, P. Soucek, Y. Caraco, F. P. Guengerich, A. J. J. Wood, Biochem. Pharm. 1997, 5, 111.

Table 7. Main signals in FAB MS mass spectra of colchiceine complexes 3-8

\begin{tabular}{|c|c|c|c|c|}
\hline Complex & Col- $\mathrm{M}^{+}(\%)$ & Col-2 $\mathrm{M}^{+} \mathrm{X}^{-}(\%)$ & $2 \mathrm{Col}^{-\mathrm{M}^{+}}(\%)$ & $2 \mathrm{Col}-2 \mathrm{M}^{+} \mathrm{X}^{-}(\%)$ \\
\hline 3 & $392(100)$ & - & $778(5)$ & - \\
\hline 4 & $408(90)$ & - & 794 (4) & - \\
\hline 5 & $424(88)$ & - & - & - \\
\hline 6 & 392 (98) & $498(10)$ & $778(5)$ & $884(5)$ \\
\hline 7 & $408(85)$ & - & 794 (3) & - \\
\hline 8 & $424(80)$ & - & $810(2)$ & - \\
\hline
\end{tabular}


[15] M. Mourelle, R. Fraginals, L. Rodrigez, L. Favari, V. Perez-Alvarez, Life Sci. 1989, 45, 891.

[16] M. F. Mackay, R. W. Gable, J. D. Morrison, L. O. Satzke, Austr. J. Chem. 1999, 52, 333.

[17] M. Schonharting, G. Mende, G. Siebert, Z. HoppeSeyler's, Physiol. Chem. 1974, 355, 1391.

[18] M. Modriansky, Y. Y. Tyurina, V. A. Tyurin, T. Matsura, A. A. Shvedova, J. C. Yalowich, Toxicology 2002, 177, 105.

[19] M. Rodriguez, J. Cerbon-Ambriz, M. L. Munoz, Arch. Med. Res. 1998, 29, 109.

[20] A. A. Nava-Ocampo, S. Suster, P. Muriel, Europ. J. Clin. Invest. 1997, 27, 77.

[21] P. Muriel, O. R. Suarez, J. Appl. Toxicol. 1994, 14, 423.

[22] R. C. Schnell, G. Vali, J. Atmos. Sci. 1976, 33, 1554.

[23] Z. Dvoraka, J. Ulrichovaa, L. Pichard-Garciab, M. Modrianskya, P. Maurelb, Toxicol. in Vitro 2002, 16, 219.

[24] S. B. Hastie, T. L. Macdonald, Biochem. Pharmacol. 1990, 39, 1271.

[25] J. Kurek, Wł. Boczoń, P. Przybylski, B. Brzeziński, J. Mol. Struct. 2007, 846, 13.
[26] J. W. Cook, J. D. Loudon, in: R. H. Manske (Ed.), The alkaloids, Vol. 2, Academic Press, New York, 1952, p. 261.

[27] J. Elguero, R. N. Muller, A. Blade-Font, R. Faure, E. J. Vincent, Bull. Soc. Chim. Belg. 1980, 89, 193.

[28] B. Gierczyk, G. Schroeder, P. Przybylski, B. Brzeziński, F. Bartl, G.Zundel, J. Mol. Struct. 2005, 738, 261.

[29] A. Huczyński, P. Przybylski, B. Brzeziński, F. Bartl, Biopolymers 2006, 81, 282.

[30] A. Huczyński, P. Przybylski, B. Brzeziński, F. Bartl, Biopolymers 2006, 82, 491.

[31] A. Huczyński, P. Przybylski, B. Brzeziński, F. Bartl, J. Phys. Chem. B 2006, 110, 15615.

[32] A. Huczyński, D. Michalak, P. Przybylski, B. Brzeziński, F. Bartl, J. Mol. Struct. 2006, 797, 99.

[33] G. P. Scott, D. S Tarbell, J. Amer. Chem. Soc. 1950, 72, 240.

[34] D. J. Morrrison, Acta Cryst. 1951, 4, 69.

[35] Y. Chen, Y.-H. Zhang, L.-J. Zhao, Phys. Chem. Chem. Phys. 2004, 6, 537.

[36] D. L. Lewis, E. D. Esters, D. J. Hodgson, J. Cryst. Mol. Struct. 1975, 5, 67. 


\section{Supporting Information}

\section{Colchiceine Complexes with Lithium, Sodium and Potassium Salts - Spectroscopic Studies}

Joanna Kurek, Piotr Barczyński

Figures of FT-IR spectra

Figure 1S. FT IR spectra (KBr) of colchiceine $\mathbf{2}$ and its complexes with lithium iodide $\mathbf{3}$, sodium iodide 4 and potassium iodide 5 at the range $3750-2500 \mathrm{~cm}^{-1}$

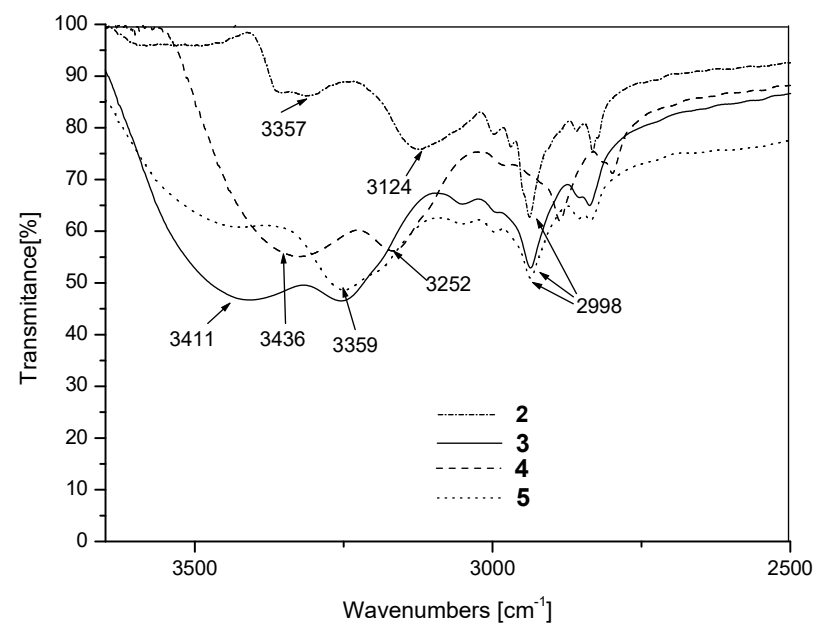

Figure 2S. FT IR spectra (nujol/fluorolub) of colchiceine complex with lithium perchlorate 6 at the range $4000-400 \mathrm{~cm}^{-1}$

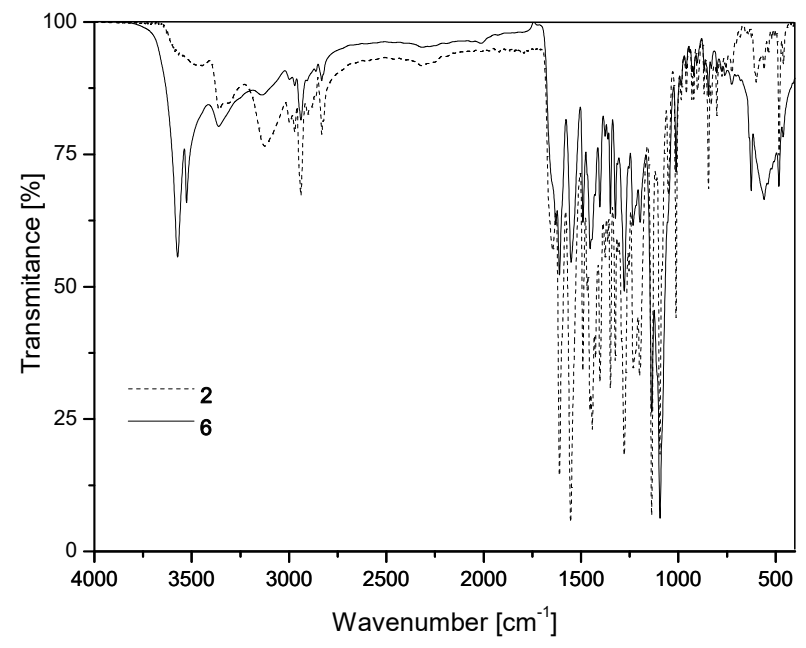


Figure 3S. FT IR spectra (nujol/fluorolub) of Colchiceine Complexes with lithium perchlorate 6 in the region of $3750-2750 \mathrm{~cm}^{-1}$

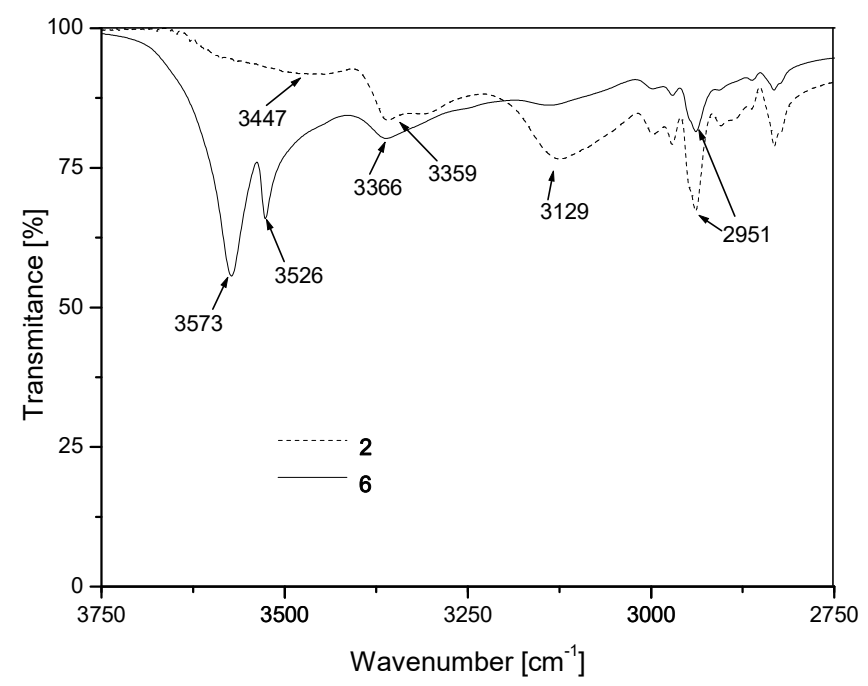


Figure 4S. FT IR spectra of Colchiceine 2 and its Complex with lithium perchlorate 6 $\left(\mathrm{CD}_{3} \mathrm{CN}\right)$ : a) $4000-500 \mathrm{~cm}^{-1}$, b) $3750-2750 \mathrm{~cm}^{-1}$

a)

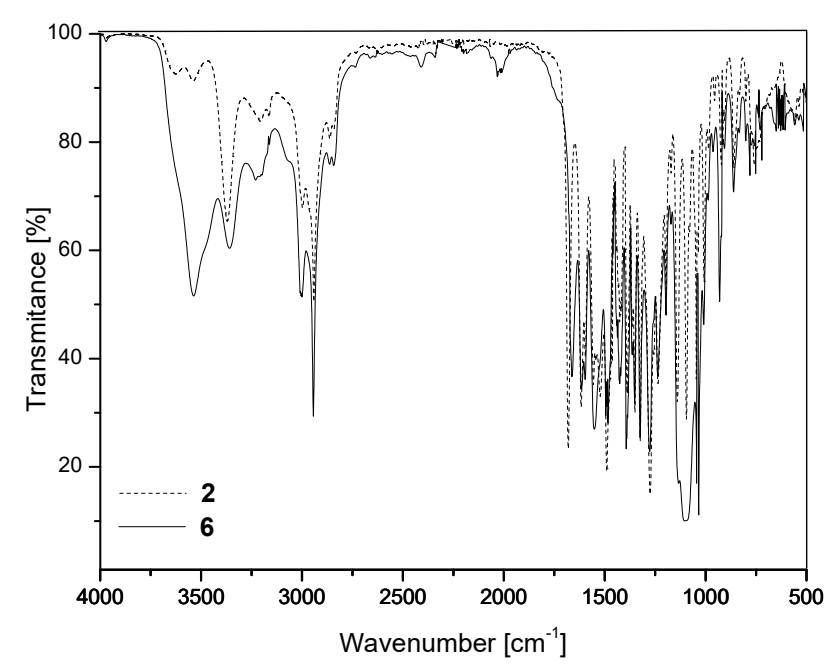

b)

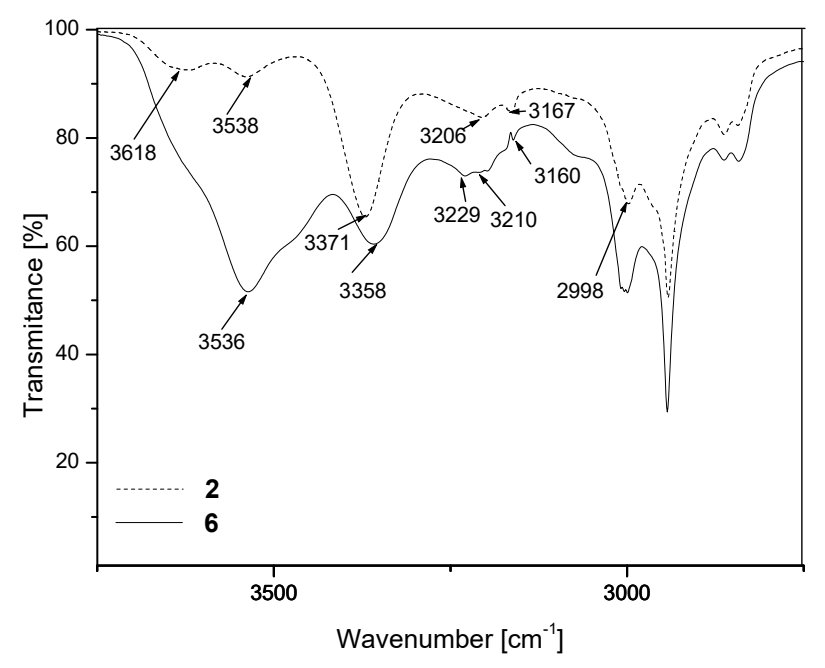

